\title{
A Predator-Prey Model in the Chemostat with Time Delay
}

\author{
Guihong Fan ${ }^{1,2}$ and Gail S. K. Wolkowicz ${ }^{1}$ \\ ${ }^{1}$ Department of Mathematics and Statistics, McMaster University, Hamilton, ON, Canada L8S 4K1 \\ ${ }^{2}$ Department of Mathematics and Statistics, York University, 4700 Keele Street, Toronto, \\ ON, Canada M3J 1P3
}

Correspondence should be addressed to Gail S. K. Wolkowicz, wolkowic@mcmaster.ca

Received 1 November 2009; Accepted 11 January 2010

Academic Editor: Yuri V. Rogovchenko

Copyright (c) 2010 G. Fan and G. S. K. Wolkowicz. This is an open access article distributed under the Creative Commons Attribution License, which permits unrestricted use, distribution, and reproduction in any medium, provided the original work is properly cited.

The aim of this paper is to study the dynamics of predator-prey interaction in a chemostat to determine whether including a discrete delay to model the time between the capture of the prey and its conversion to viable biomass can introduce oscillatory dynamics even though there is a globally asymptotically stable equilibrium when the delay is ignored. Hence, Holling type I response functions are chosen so that no oscillatory behavior is possible when there is no delay. It is proven that unlike the analogous model for competition, as the parameter modeling the delay is increased, Hopf bifurcations can occur.

\section{Introduction}

The chemostat, also known as a continuous stir tank reactor (CSTR) in the engineering literature, is a basic piece of laboratory apparatus used for the continuous culture of microorganisms. It has potential applications for such processes as wastewater decomposition and water purification. Some ecologists consider it a lake in a laboratory. It can be thought of as three vessels, the feed bottle that contains fresh medium with all the necessary nutrients, the growth chamber where the microorganisms interact, and the collection vessel. The fresh medium from the feed bottle is continuously added to the growth chamber. The growth chamber is well stirred and its contents are then removed to the collection vessel at a rate that maintains constant volume. For a detailed description of the importance of the chemostat and its application in biology and ecology, one can refer to [1,2].

The following system describes a food chain in the chemostat where a predator population feeds on a prey population of microorganisms that in turn consumes a 
nonreproducing nutrient that is assumed to be growth limiting at low concentrations

$$
\begin{aligned}
& \dot{s}(t)=\left(s^{0}-s(t)\right) D_{0}-\frac{x(t) f(s(t))}{\eta}, \\
& \dot{x}(t)=x(t)(-D+f(s(t)))-\frac{y(t) g(x(t))}{\xi}, \\
& \dot{y}(t)=-\Delta y(t)+y(t) g(x(t)) .
\end{aligned}
$$

Here $s(t)$ represents the concentration of the growth limiting nutrient, $x(t)$ the density of the prey population, and $y(t)$ the density of the predator population. Parameter $s^{0}$ denotes the concentration of the growth limiting nutrient in the feed vessel, $D_{0}$ the dilution rate, $\eta(\xi)$ the growth yield constant, $D(\Delta)$ the sum of the dilution rate $D_{0}$ and the natural species specific death rate of the prey (predator) population, respectively. Here $f(s)$ denotes the functional response of the prey population on the nutrient and $g(x)$ denotes the functional response of the predator on the prey.

Butler et al. [3] considered the coexistence of two competing predators feeding on a single prey population growing in the chemostat. As a subsystem of their model, they studied the global stability of system (1.1) with both $f(s)$ and $g(x)$ taking the form of Holling type II. They proved that under certain conditions the interior equilibrium is globally asymptotically stable with respect to the interior of the positive cone. However, they also proved that for certain ranges of the parameters there is at least one nontrivial limit cycle and conjectured that the limit cycle is unique and would be a global attractor with respect to the noncritical orbits in the open positive octant. This conjecture was partially solved by Kuang [4]. He showed that there is a range of parameters for which a unique periodic orbit exists and roughly located the position of the limit cycle.

Bulter and Wolkowicz [5] studied predator mediated coexistence in the chemostat assuming $D_{0}=D=\Delta$. Model (1.1) was studied as a submodel. For general monotone response functions, Bulter and Wolkowicz showed that (1.1) is uniformly persistent if the sum of the break even concentrations of substrate and prey is less than the input rate of the nutrient $s^{0}$. However they showed that it is necessary to specify the form of the response functions in order to discuss the global dynamics of the model. If $f(s)$ is modelled by Holling type I or II and $g(x)$ by Holling type I, Bulter and Wolkowicz proved that (1.1) could have up to three equilibrium points and that there is a transfer of global stability from one equilibrium point to another as different parameters are varied making conditions favorable enough for a new population to survive. In this case, there are no periodic solutions. However, even if $f(s)$ is given by Holling type I, if $g(x)$ is given by Holling type II, they showed that a Hopf bifurcation can occur in (1.1), and numerical simulations indicated that the bifurcating periodic solution was asymptotically stable.

We include a time delay in (1.1) to model the time between the capture of the prey and its conversion to viable biomass. Our aim is to show that such a delay can induce nontrivial periodic solutions in a model where there is always a globally asymptotically stable equilibrium when delay is ignored, and hence no such periodic solutions are possible otherwise. For this reason we select the response functions of the simplest form; that is, we choose the Holling type I form for both $f(s)$ and $g(x)$, so that (1.2) always has a globally asymptotically stable equilibrium when the conversion process is assumed to occur instantaneously. It is interesting to note that in the analogous model of competition between 
two species in the chemostat, delay cannot induce oscillatory behavior for any reasonable monotone response functions (see Wolkowicz and Xia [6]).

With delay modelling the time required for the predator to process the prey after it has been captured, the model is given by

$$
\begin{aligned}
& \dot{s}(t)=\left(s^{0}-s(t)\right) D_{0}-\frac{x(t) f(s(t))}{\eta}, \\
& \dot{x}(t)=x(t)(-D+f(s(t)))-\frac{y(t) g(x(t))}{\xi}, \\
& \dot{y}(t)=-\Delta y(t)+e^{-\Delta \tau} y(t-\tau) g(x(t-\tau)) .
\end{aligned}
$$

For $t \in[-\tau, 0]$,

$$
s(0)=s_{0} \in \operatorname{int} \mathbb{R}_{+}, \quad(x(t), y(t))=(\phi, \psi) \in \mathbb{C}\left([-\tau, 0], \text { int } \mathbb{R}_{+}^{2}\right) .
$$

Here variables $s(t), x(t), y(t)$, and parameters $s^{0}, D_{0}, \eta, \xi, D_{0}, D$, and $\Delta$ have the same interpretation as for model (1.1). Note therefore that $D \geqslant D_{0}$, and $\Delta \geqslant D_{0}$. The additional parameter $\tau$ is a nonnegative constant modelling the time required for the conversion process. Hence, $e^{-\Delta \tau} y(t-\tau)$ represents the concentration of the predator population in the growth chamber at time $t$ that were available at time $t-\tau$ to capture prey and were able to avoid death and washout during the $\tau$ units of time required to process the captured prey.

We analyze the stability of each equilibrium and prove that the coexistence equilibrium can undergo Hopf bifurcations. Numerical simulations appear to show that (1.2) can have a stable periodic solution bifurcating from the coexistence equilibrium as the delay parameter increases from zero. This periodic orbit can then disappear through a secondary Hopf bifurcation as the delay parameter increases further.

\section{Scaling of the Model and Existence of Solutions}

Suppose that functions $f(s)$ and $g(s)$ are of Holling type I form, that is, $f(s)=\alpha s(\alpha>0)$ and $g(x)=k x(k>0)$. System (1.2) reduces to

$$
\begin{aligned}
& \dot{s}(t)=\left(s^{0}-s(t)\right) D_{0}-\frac{\alpha x(t) s(t)}{\eta}, \\
& \dot{x}(t)=x(t)(-D+\alpha s(t))-\frac{k x(t) y(t)}{\xi}, \quad t>0, \\
& \dot{y}(t)=-\Delta y(t)+k e^{-\Delta \tau} y(t-\tau) x(t-\tau) .
\end{aligned}
$$


Introducing the following change of variables gives:

$$
\begin{aligned}
& \breve{t}=D_{0} t, \quad \breve{s}(\breve{t})=\frac{s(t)}{s^{0}}, \quad \breve{x}(\breve{t})=\frac{x(t)}{s^{0} \eta}, \quad \breve{y}(\breve{t})=\frac{y(t)}{\xi s^{0} \eta}, \\
& \breve{\tau}=D_{0} \tau, \quad \breve{D}=\frac{D}{D_{0}}, \quad \breve{\Delta}=\frac{\Delta}{D_{0}}, \quad \breve{k}=\frac{k s^{0} \eta}{D_{0}}, \quad \breve{\alpha}=\frac{\alpha s^{0}}{D_{0}}, \\
& \frac{\mathrm{d} \breve{s}(\breve{t})}{\mathrm{d} \breve{t}}=\frac{1}{s^{0}} \frac{\mathrm{d} s(t)}{\mathrm{d} t} \frac{\mathrm{d} t}{\mathrm{~d} \breve{t}}=\frac{1}{s^{0} D_{0}} \frac{\mathrm{d} s(t)}{\mathrm{d} t} \\
& =\frac{1}{s^{0} D_{0}}\left(\left(s^{0}-s(t)\right) D_{0}-\frac{\alpha x(t) s(t)}{\eta}\right) \\
& =1-\frac{s(t)}{s^{0}}-\frac{\alpha s^{0}}{D_{0}} \frac{x(t)}{s^{0} \eta} \frac{s(t)}{s^{0}} \\
& =1-\breve{s}(\breve{t})-\breve{\alpha} \breve{x}(\breve{t}) \breve{s}(\breve{t}), \\
& \frac{\mathrm{d} \breve{x}(\breve{t})}{\mathrm{d} \breve{t}}=\frac{1}{s^{0} \eta} \frac{\mathrm{d} x(t)}{\mathrm{d} t} \frac{\mathrm{d} t}{\mathrm{~d} \breve{t}}=\frac{1}{s^{0} \eta D_{0}} \frac{\mathrm{d} x(t)}{\mathrm{d} t} \\
& =\frac{1}{s^{0} \eta D_{0}}\left(x(t)(-D+\alpha s(t))-\frac{k x(t) y(t)}{\xi}\right) \\
& =\frac{x(t)}{s^{0} \eta}\left(-\frac{D}{D_{0}}+\frac{\alpha s^{0}}{D_{0}} \frac{s(t)}{s^{0}}\right)-\frac{k s^{0} \eta}{D_{0}} \frac{x(t)}{s^{0} \eta} \frac{y(t)}{s^{0} \eta \xi} \\
& =\breve{x}(\breve{t})(-\breve{D}+\breve{\alpha} \breve{s}(\breve{t}))-\breve{k} \breve{x}(\breve{t}) \breve{y}(\breve{t}), \\
& \frac{\mathrm{d} \breve{y}(\breve{t})}{\mathrm{d} \breve{t}}=\frac{1}{s^{0} \eta \xi} \frac{\mathrm{d} y(t)}{\mathrm{d} t} \frac{\mathrm{d} t}{\mathrm{~d} \breve{t}}=\frac{1}{s^{0} \eta \xi D_{0}} \frac{\mathrm{d} y(t)}{\mathrm{d} t} \\
& =\frac{1}{s^{0} \eta \xi D_{0}}\left(-\Delta y(t)+k e^{-\Delta \tau} y(t-\tau) x(t-\tau)\right) \\
& =\frac{-\Delta y(t)}{s^{0} \eta \xi D_{0}}+\frac{k e^{-\Delta \tau}}{s^{0} \eta \xi D_{0}} y(t-\tau) x(t-\tau) \\
& =\frac{-\Delta}{D_{0}} \frac{y(t)}{s^{0} \eta \xi}+\frac{k s^{0} \eta}{D_{0}} e^{-\left(\Delta / D_{0}\right) D_{0} \tau} \frac{y(t-\tau)}{s^{0} \eta \xi} \frac{x(t-\tau)}{s^{0} \eta} \\
& =-\breve{\Delta} \breve{y}(\breve{t})+\breve{k} e^{-\breve{\Delta} \breve{y}} \breve{y}(\breve{t}-\breve{\tau}) \breve{x}(\breve{t}-\breve{\tau}) \text {. }
\end{aligned}
$$

With this change of variables, omitting the "s for convenience, system (2.1) becomes

$$
\begin{aligned}
& \dot{s}(t)=1-s(t)-\alpha x(t) s(t), \\
& \dot{x}(t)=x(t)(-D+\alpha s(t))-k y(t) x(t), \\
& \dot{y}(t)=-\Delta y(t)+k e^{-\Delta \tau} y(t-\tau) x(t-\tau),
\end{aligned}
$$


where $\Delta \geqslant 1$ and $D \geqslant 1$, with initial data given by (1.3). For biological significance, a point is assumed to be an equilibrium point of (2.3) only if all of its components are nonnegative.

Let $\tau=0$. Model (2.3) reduces to a special case of the model considered in [7]. If $D>\alpha$, the model has only one equilibrium point $(1,0,0)$ and it is globally asymptotically stable. If $D<\alpha$ and $1-D / \alpha-\Delta D / k<0$, the model has a second equilibrium point $(D / \alpha,(\alpha-D) / \alpha D, 0)$ and it is globally asymptotically stable. When $1-D / \alpha-\Delta D / k>0$, the model has a third equilibrium point $(k /(k+\alpha \Delta), \Delta / k, \alpha /(k+\alpha D)-D / k)$ and it is the global attractor. Therefore, model (2.3) has no periodic solutions when the time delay is ignored. If $g(x)$ is of Holling type II form, Butler and Wolkowicz [5] proved that a Hopf bifurcation is possible resulting in a periodic solution for a certain range of parameter values. We emphasize again here, that it is for this reason that in this paper we restrict our attention to the simplest case for both response functions, that is, Holling type I, in order to see whether delay can be responsible for periodic solutions in (1.2).

Theorem 2.1. Assuming $\left(s_{0}, \phi(\theta), \psi(\theta)\right) \in \operatorname{int} \mathbb{R}_{+} \times \mathbb{C}\left([-\tau, 0]\right.$, int $\left.\mathbb{R}_{+}^{2}\right)$, then there exists a unique solution $(s(t), x(t), y(t))$ of (2.3) passing through $\left(s_{0}, \phi(\theta), \psi(\theta)\right)$ with $s(t)>0, x(t)>0$ and $y(t)>0$ for $t \in[0, \infty)$. The solution is bounded. In particular, given any $\epsilon_{0}>0, x(t)<1+\epsilon_{0}$ for all sufficiently large $t$.

Proof. For $t \in[0, \tau]$, one has $t-\tau \in[-\tau, 0], x(t-\tau)=\phi(t-\tau)$, and $y(t-\tau)=\psi(t-\tau)$. System (2.3) becomes

$$
\begin{aligned}
& \dot{s}(t)=1-s(t)-\alpha x(t) s(t), \\
& \dot{x}(t)=x(t)(-D+\alpha s(t))-k y(t) x(t), \\
& \dot{y}(t)=-\Delta y(t)+k e^{-\Delta \tau} \phi(t-\tau) \psi(t-\tau),
\end{aligned}
$$

a system of nonautonomous ordinary differential equations with initial conditions $s(0)=s_{0}$, $x(0)=\phi(0)$, and $y(0)=\psi(0)$. Since the right-hand side of (2.4) is differentiable in both $x$ and $y$, by Theorems 2.3, 3.1, and Corollary 4.3 in Miller and Michel [8], there exists a unique solution defined on $[0, \tau]$ satisfying (2.4). By using the method of steps in Bellman and Cooke [9], it can be shown that the solution through $\left(s_{0}, \phi(\theta), \psi(\theta)\right)$ is defined for all $t \geqslant 0$.

Now we prove $s(t)>0$ for all $t>0$. From the first equation of (2.3),

$$
\dot{s}(t)=1-s(t)-\alpha x(t) s(t)
$$

Proceed using the method of contradiction. Suppose that there exists a first $t^{\star}$ such that $s\left(t^{\star}\right)=$ 0 and $s(t)>0$ for $t \in\left[0, t^{*}\right)$. Then $\dot{s}\left(t^{\star}\right) \leqslant 0$. But from the first equation of (2.3)

$$
\dot{s}\left(t^{\star}\right)=1-s\left(t^{\star}\right)-\alpha x\left(t^{\star}\right) s\left(t^{\star}\right)=1>0,
$$

a contradiction. 
To prove $x(t)>0$ for $t \in[0, \infty)$, assume there is a first $\bar{t}>0$ such that $x(\bar{t})=0$, and $x(t)>0$ for $t \in[0, \bar{t})$. Divide both sides of the second equation of $(2.3)$ by $x(t)$ and integrate from 0 to $\bar{t}$, to obtain

$$
x(\bar{t})=\phi(0) \exp \left(\int_{0}^{\bar{t}}(-D+\alpha s(t)-k y(t)) \mathrm{d} t\right)>0,
$$

contradicting $x(\bar{t})=0$.

To show that $y(t)$ is positive on $[0, \infty)$, suppose that there exists $t^{\star}>0$ such that $y\left(t^{\star}\right)=$ 0 , and $y(t)>0$ for $t \in\left[0, t^{*}\right)$. Then $\dot{y}\left(t^{\star}\right) \leqslant 0$. From the third equation of $(2.3)$, we have

$$
\begin{aligned}
\dot{y}\left(t^{\star}\right) & =-\Delta y\left(t^{\star}\right)+k e^{-\Delta \tau} y\left(t^{\star}-\tau\right) x\left(t^{\star}-\tau\right) \\
& =k e^{-\Delta \tau} y\left(t^{\star}-\tau\right) x\left(t^{\star}-\tau\right)>0,
\end{aligned}
$$

a contradiction.

To prove the boundedness of solutions, define

$$
\omega(t)=s(t)+x(t)+e^{\Delta \tau} y(t+\tau)-1, \quad \text { for } t \geqslant 0 .
$$

It follows that

$$
\begin{aligned}
\dot{\omega}(t) & =1-s(t)-D x(t)-\Delta e^{\Delta \tau} y(t+\tau) \\
& \leqslant 1-s(t)-x(t)-e^{\Delta \tau} y(t+\tau) \\
& \leqslant-\omega(t),
\end{aligned}
$$

where the first inequality holds since $D \geqslant 1, \Delta \geqslant 1, x(t)>0$ and $y(t+\tau)>0$. It follows that

$$
s(t)+x(t)+e^{\Delta \tau} y(t+\tau) \leqslant 1+\left(s_{0}+x(0)+e^{\Delta \tau} y(\tau)-1\right) e^{-t} \longrightarrow 1 \quad \text { as } t \longrightarrow \infty
$$

Therefore, the solution $(s(t), x(t), y(t))$ is bounded, and given any $\epsilon_{0}>0, x(t)<1+\epsilon_{0}$ for all sufficiently large $t$.

\section{Equilibria and Stability}

Model (2.3) has three equilibrium points: $E_{1}=(1,0,0), E_{2}=(D / \alpha,(\alpha-D) / \alpha D, 0)$, and

$$
E_{+}=\left(s_{+}(\tau), x_{+}(\tau), y_{+}(\tau)\right)=\left(\frac{1}{1+(\alpha \Delta / k) e^{\Delta \tau}}, \frac{\Delta}{k} e^{\Delta \tau}, \frac{\alpha}{k+\alpha \Delta e^{\Delta \tau}}-\frac{D}{k}\right) .
$$

We call $E_{1}$ the washout equilibrium, $E_{2}$ the single species equilibrium, and $E_{+}$the coexistence equilibrium. For the sake of biological significance, $E_{+}$exists (distinct from $E_{2}$ ) if and only if 
its third coordinate $y_{+}(\tau)=\left(\alpha s_{+}(\tau)-D\right) / k>0$, that is, $s_{+}(\tau)>D / \alpha$, or equivalently, $\tau$ lies between 0 and $\tau_{c}$, where

$$
\tau_{c}=\frac{1}{\Delta} \ln \left(\frac{k}{\Delta}\left(\frac{1}{D}-\frac{1}{\alpha}\right)\right)
$$

Note that if $(k / \Delta)(1 / D-1 / \alpha) \leqslant 1$, the equilibrium $E_{+}$does not exist for any $\tau(\geqslant 0)$, and if $(k / \Delta)(1 / D-1 / \alpha)=1$, then $E_{+}=E_{2}$.

The linearization of (2.3) about an equilibrium $(s, x, y)$ is given by

$$
\begin{aligned}
{\left[\begin{array}{l}
\dot{z}_{1}(t) \\
\dot{z}_{2}(t) \\
\dot{z}_{3}(t)
\end{array}\right]=} & {\left[\begin{array}{ccc}
-1-\alpha x & -\alpha s & 0 \\
\alpha x & -D+\alpha s-k y & -k x \\
0 & 0 & -\Delta
\end{array}\right]\left[\begin{array}{l}
z_{1}(t) \\
z_{2}(t) \\
z_{3}(t)
\end{array}\right] } \\
& +\left[\begin{array}{ccc}
0 & 0 & 0 \\
0 & 0 & 0 \\
0 & k e^{\Delta \tau} y & k e^{\Delta \tau} x
\end{array}\right]\left[\begin{array}{l}
z_{1}(t-\tau) \\
z_{2}(t-\tau) \\
z_{3}(t-\tau)
\end{array}\right] .
\end{aligned}
$$

The associated characteristic equation is given by

$$
\operatorname{det}\left[\begin{array}{ccc}
-1-\alpha x-\lambda & -\alpha s & 0 \\
\alpha x & -D+\alpha s-k y-\lambda & -k x \\
0 & k e^{-\Delta \tau-\lambda \tau} y & -\Delta+k e^{-\Delta \tau-\lambda \tau} x-\lambda
\end{array}\right]=0 .
$$

Direct calculation of the left-hand side of (3.4) gives

$$
\begin{aligned}
(-\Delta+ & \left.k e^{-(\Delta+\lambda) \tau} x-\lambda\right)\left\{(-1-\alpha x-\lambda)(-D+\alpha s-k y-\lambda)+\alpha^{2} s x\right\} \\
& +k x k e^{-(\Delta+\lambda) \tau} y(-1-\alpha x-\lambda) \\
= & (-\Delta-\lambda)\left\{(1+\alpha x+\lambda)(D-\alpha s+k y+\lambda)+\alpha^{2} s x\right\}+e^{-(\Delta+\lambda) \tau} k x \\
& \times\left\{k y(-1-\alpha x-\lambda)+(1+\alpha x+\lambda)(D-\alpha s+k y+\lambda)+\alpha^{2} s x\right\} \\
= & (-\Delta-\lambda)\left\{(1+\alpha x+\lambda)(D-\alpha s+k y+\lambda)+\alpha^{2} s x\right\} \\
& +e^{-(\Delta+\lambda) \tau} k x\left\{(1+\alpha x+\lambda)(D-\alpha s+\lambda)+\alpha^{2} s x\right\} \\
= & (-\Delta-\lambda)\{(\lambda+1)(\lambda+D+k y)+\alpha x(\lambda+D+k y)-\alpha s(\lambda+1)\} \\
& +e^{-(\Delta+\lambda) \tau} k x\{(\lambda+1)(\lambda+D)+\alpha x(\lambda+D)-\alpha s(\lambda+1)\} .
\end{aligned}
$$


For convenience, define

$$
\begin{aligned}
P(\lambda):= & (-\Delta-\lambda)\{(\lambda+1)(\lambda+D+k y)+\alpha x(\lambda+D+k y)-\alpha s(\lambda+1)\} \\
& +e^{-(\Delta+\lambda) \tau} k x\{(\lambda+1)(\lambda+D)+\alpha x(\lambda+D)-\alpha s(\lambda+1)\} .
\end{aligned}
$$

Theorem 3.1. Equilibrium $E_{1}$ is stable if $\alpha<D$ and unstable if $\alpha>D$.

Proof. Evaluating the characteristic equation at $E_{1}$ gives

$$
\left.P(\lambda)\right|_{E_{1}}=-(\Delta+\lambda)(\lambda+1)(\lambda+D-\alpha)=0 .
$$

The eigenvalues -1 and $-\Delta$ are both negative. The third eigenvalue is $-D+\alpha$. Therefore the equilibrium $E_{1}$ is stable if $\alpha<D$ and unstable if $\alpha>D$.

Remark 3.2. If $\alpha<D$, then there is only one equilibrium, $E_{1}$. If $\alpha>D$, equilibrium $E_{2}$ also exists.

Lemma 3.3. Assume $\alpha>D$. The characteristic equation evaluated at $E_{2}$ has two negative eigenvalues, and the remaining eigenvalues are solutions of

$$
(\lambda+\Delta) e^{(\lambda+\Delta) \tau}=k\left(\frac{1}{D}-\frac{1}{\alpha}\right)
$$

In addition, the characteristic equation evaluated at $E_{2}$ has zero as an eigenvalue if and only if $\tau=\tau_{c}$.

Proof. Assume $\alpha>D$. Equilibrium $E_{2}$ exists. Consider the characteristic equation at $E_{2}$. Since $(\alpha-D) / \alpha D=(1-s) / \alpha s$ at $E_{2}$

$$
\begin{aligned}
\left.P(\lambda)\right|_{E_{2}}= & \{(\lambda+1)(\lambda+D)+\alpha x(\lambda+D)-\alpha s(\lambda+1)\} \\
& \times\left(-\lambda-\Delta+e^{-(\Delta+\lambda) \tau} k x\right) \\
= & \left\{(\lambda+1)(\lambda+D)+\frac{1-s}{s}(\lambda+D)-D(\lambda+1)\right\} \\
& \times\left(-\lambda-\Delta+e^{-(\Delta+\lambda) \tau} k \frac{\alpha-D}{\alpha D}\right) \\
= & \left\{\lambda(\lambda+1)-(\lambda+D)+\frac{\lambda+D}{s}\right\}\left(-\lambda-\Delta+k \frac{\alpha-D}{\alpha D} e^{-(\Delta+\lambda) \tau}\right) \\
= & -\left(\lambda^{2}+\frac{\alpha}{D} \lambda+\alpha-D\right)\left(\lambda+\Delta-k \frac{\alpha-D}{\alpha D} e^{-(\Delta+\lambda) \tau}\right) \\
= & -e^{-(\Delta+\lambda) \tau}\left(\lambda-\lambda_{1}\right)\left(\lambda-\lambda_{2}\right)\left((\lambda+\Delta) e^{(\Delta+\lambda) \tau}-k\left(\frac{1}{D}-\frac{1}{\alpha}\right)\right)=0,
\end{aligned}
$$

where $\lambda_{1}+\lambda_{2}=-\alpha / D$ and $\lambda_{1} \lambda_{2}=\alpha-D>0$. Therefore, $\lambda_{1}$ and $\lambda_{2}$ have negative real parts. The rest of the eigenvalues are roots of (3.8). 
Assuming that $\lambda=0$ is a root of (3.8), we have

$$
\Delta e^{\Delta \tau}=k\left(\frac{1}{D}-\frac{1}{\alpha}\right)
$$

Solving for $\tau$ gives

$$
\tau=\frac{1}{\Delta} \ln \left(\frac{k}{\Delta}\left(\frac{1}{D}-\frac{1}{\alpha}\right)\right)=\tau_{c}
$$

Theorem 3.4. Assume that $D \geqslant 1, \Delta \geqslant 1, k>0, \alpha>0$, and $(k / \Delta)(1 / D-1 / \alpha) \geqslant 1$ so that $\tau_{c} \geqslant 0$. Equilibrium $E_{2}$ is locally asymptotically stable if $\tau>\tau_{c}$ and unstable if $\tau<\tau_{c}$. If $D=1$, then equilibrium $E_{2}$ is globally asymptotically stable for $\tau>(1 / \Delta) \ln (k / \Delta)$.

Proof. Assume that $\tau>\tau_{c}$. Assumptions $k>0, \Delta \geqslant 1$, and $(k / \Delta)(1 / D-1 / \alpha) \geqslant 1$ imply $1 / D>1 / \alpha$, or equivalently $\alpha>D$. By Lemma 3.3, to prove that equilibrium $E_{2}$ is locally asymptotically stable, one only needs to show that (3.8) admits no root with nonnegative real part.

Consider the real roots of (3.8) first. Note that $1 / D>1 / \alpha$. Equation (3.8) has no solution for $\lambda \leqslant-\Delta$. Otherwise the left-hand side would be less than zero, but the right-hand side would be greater than zero. Assume $\lambda>-\Delta$. The left-hand side of (3.8) is a monotone increasing function in both $\lambda$ and $\tau$, takes value 0 at $\lambda=-\Delta$, and goes to positive infinity as $\lambda \rightarrow+\infty$ or $\tau \rightarrow+\infty$. By Lemma 3.3, when $\tau=\tau_{c}$, then $\lambda=0$ is a solution of (3.8). Thus for $\tau>\tau_{c}$, any real root $\lambda$ of (3.8) must satisfy $-\Delta<\lambda<0$.

For any $\tau=\tilde{\tau}<\tau_{c}$, we have $\left.(\lambda+\Delta) e^{(\lambda+\Delta) \tau}\right|_{\tau=\tilde{\tau}, \lambda=0}<k(1 / D-1 / \alpha)$ and $\lim _{\lambda \rightarrow+\infty}(\lambda+$ $\Delta) e^{(\lambda+\Delta) \tilde{\tau}}=+\infty$. Therefore there exists at least one $\lambda=\tilde{\lambda}>0$ such that $(\tilde{\tau}, \tilde{\lambda})$ is a solution of (3.8). Equilibrium $E_{2}$ is unstable if $\tau<\tau_{c}$.

In what follows, we prove that if $\tau>\tau_{c}$ all complex eigenvalues of (3.8) have negative real parts. Suppose that $\lambda+\Delta=\gamma+i \beta \quad(\beta>0)$ is a solution of (3.8). Using the Euler formula, we have

$$
\gamma \cos (\beta \tau)-\beta \sin (\beta \tau)+i(\gamma \sin (\beta \tau)+\beta \cos (\beta \tau))=k\left(\frac{1}{D}-\frac{1}{\alpha}\right) e^{-\gamma \tau} .
$$

Equating the real parts and imaginary parts of the equation, we have

$$
\begin{gathered}
\gamma \cos (\beta \tau)-\beta \sin (\beta \tau)=k\left(\frac{1}{D}-\frac{1}{\alpha}\right) e^{-\gamma \tau} \\
\gamma \sin (\beta \tau)+\beta \cos (\beta \tau)=0 .
\end{gathered}
$$

Squaring both equations, adding, and taking the square root on both sides give

$$
\sqrt{r^{2}+\beta^{2}} e^{r \tau}=k\left(\frac{1}{D}-\frac{1}{\alpha}\right)
$$


The left-hand side of (3.14) is monotonically increasing in $\gamma, \beta$, and $\tau$ provided that $\gamma>0$. Since (3.14) has solution $\gamma=\Delta, \beta=0$ at $\tau=\tau_{c}$, any roots of (3.14) must satisfy $\gamma<\Delta$ since $\tau>\tau_{c}$. Hence $\operatorname{Re}\{\lambda\}=\gamma-\Delta<0$. Therefore (3.8) has no complex eigenvalue with nonnegative real part and so $E_{2}$ is locally asymptotically stable for $\tau>\tau_{c}$.

Assume that $D=1$. Now we prove that $E_{2}$ is globally asymptotically stable when $\tau>(1 / \Delta) \ln (k / \Delta)$, or equivalently $k e^{-\Delta \tau}<\Delta$. In this case, choose $\epsilon_{0}>0$ small enough such that $k e^{-\Delta \tau}\left(1+\epsilon_{0}\right)<\Delta$. By Theorem 2.1, for such $\epsilon_{0}$, there exists a $T>0$ so that $0<x(t)<1+\epsilon_{0}$ for $t>T$. Hence, for $t>T+\tau, k e^{-\Delta \tau} x(t-\tau)<\Delta$. In Example 5.1 of Kuang ([10, page 32]), choose $\rho(t)=\tau, a(t)=\Delta, b(t)=k e^{-\Delta \tau} x(t-\tau)$, and $\alpha=\Delta / 2$. We obtain $\left(k e^{-\Delta \tau}\left(1+\epsilon_{0}\right)\right)^{2}<\Delta^{2}=4(\Delta-\alpha) \alpha$. Therefore $y(t) \rightarrow 0$ as $t \rightarrow \infty$. Let $z(t)=s(t)+x(t)$. Noting $D=1$, from (2.3), we have $\dot{z}(t)=1-z(t)-k x(t) y(t)$. Multiply by the integrating factor $e^{t},\left(z(t) e^{t}\right)^{\prime}=e^{t}(1-k x(t) y(t))$. Integrating both sides from 0 to $t$ gives

$$
\begin{aligned}
z(t) & =e^{-t} z(0)+e^{-t}\left(e^{t}-1\right)-e^{-t} \int_{0}^{t} e^{s} k x(s) y(s) \mathrm{d} s \\
& =1+e^{-t}(z(0)-1)-e^{-t} \int_{0}^{t} e^{s} k x(s) y(s) \mathrm{d} s .
\end{aligned}
$$

If $\lim _{t \rightarrow \infty} \int_{0}^{t} e^{s} k x(s) y(s) \mathrm{d} s<\infty$, then $\lim _{t \rightarrow \infty} e^{-t} \int_{0}^{t} e^{s} k x(s) y(s) \mathrm{d} s=0$. Therefore $\lim _{t \rightarrow \infty} z(t)=$ 1. If $\lim _{t \rightarrow \infty} \int_{0}^{t} e^{s} k x(s) y(s) \mathrm{d} s=\infty$, by L'Hôspital's rule,

$$
\begin{aligned}
\lim _{t \rightarrow+\infty} e^{-t} \int_{0}^{t} e^{s} k x(s) y(s) \mathrm{d} s & =\lim _{t \rightarrow+\infty} \frac{\int_{0}^{t} e^{s} k x(s) y(s) \mathrm{d} s}{e^{t}}=\lim _{t \rightarrow+\infty} \frac{e^{t} k x(t) y(t)}{e^{t}} \\
& =\lim _{t \rightarrow+\infty} k x(t) y(t)=0,
\end{aligned}
$$

since $x(t)$ is bounded and $\lim _{t \rightarrow \infty} y(t)=0$. It again follows that $\lim _{t \rightarrow \infty} z(t)=1$. Hence

$$
\lim _{t \rightarrow \infty} s(t)+x(t)=1
$$

We show that $\lim _{t \rightarrow \infty} s(t)=1 / \alpha$ and $\lim _{t \rightarrow \infty} x(t)=(\alpha-1) / \alpha$. First assume that the limits exist, that is, $\lim _{t \rightarrow \infty} s(t)=\bar{s}$ and $\lim _{t \rightarrow \infty} x(t)=\bar{x}$. From (2.3), we know that $\dot{s}(t)$ and $\dot{x}(t)$ are uniformly continuous since $s(t), x(t)$, and $y(t)$ are bounded. By Theorem A.3, it follows that $\lim _{t \rightarrow \infty} \dot{S}(t)=0$ and $\lim _{t \rightarrow \infty} \dot{x}(t)=0$. Note that $\lim _{t \rightarrow \infty} y(t)=0$. Letting $t \rightarrow \infty$ in (2.3) 
gives

$$
\begin{gathered}
(1-\bar{s})-\alpha \bar{x} \bar{s}=0 \\
\bar{x}(-1+\alpha \bar{s})=0 .
\end{gathered}
$$

Either $(\bar{s}, \bar{x})=(1,0)$ or $(\bar{s}, \bar{x})=(1 / \alpha,(\alpha-1) / \alpha)$. Assume that $(\bar{s}, \bar{x})=(1,0)$, that is, $\lim _{t \rightarrow \infty} s(t)=1$ and $\lim _{t \rightarrow \infty} x(t)=0$. Note that $\alpha>D$. There exists $\epsilon>0$ such that $\alpha-D-(\alpha+k) \epsilon>0$. For such $\epsilon$, there exists a sufficiently large $t$ so that $s(t)>1-\epsilon$ and $0<y(t)<\epsilon$. Recalling that $x(t)>0$, by $(2.3)$

$$
\dot{x}(t)>x(t)(-D+\alpha(1-\epsilon)-k \epsilon)=x(t)(\alpha-D-\alpha \epsilon-k \epsilon)>0,
$$

for all sufficiently large $t$. Therefore it is impossible for $x(t)$ to approach 0 from above giving a contradiction. Therefore, we must have $(\bar{s}, \bar{x})=(1 / \alpha,(\alpha-1) / \alpha)$.

Now suppose that the limits do not exist. In particular if $x(t)$ does not converge, then let $\bar{x}=\lim \sup _{t \rightarrow \infty} x(t)$ and $\underline{x}=\liminf _{t \rightarrow \infty} x(t)$. By Lemma A.2 in the appendix, there exists $\left\{t_{m}\right\} \uparrow \infty$ and $\left\{s_{m}\right\} \uparrow \infty$ such that

$$
\begin{array}{ll}
\lim _{m \rightarrow \infty} x\left(t_{m}\right)=\bar{x}, & \lim _{m \rightarrow \infty} \dot{x}\left(t_{m}\right)=0, \\
\lim _{m \rightarrow \infty} x\left(s_{m}\right)=\underline{x} & \lim _{m \rightarrow \infty} \dot{x}\left(s_{m}\right)=0 .
\end{array}
$$

From (2.3),

$$
x\left(t_{m}\right)\left(-D+\alpha s\left(t_{m}\right)+k y\left(t_{m}\right)\right)=0 .
$$

Noting that $x\left(t_{m}\right)>0$, we have $s\left(t_{m}\right)=\left(1-k y\left(t_{m}\right)\right) / \alpha$. Since $\lim _{t \rightarrow \infty} y(t)=0, \lim _{t \rightarrow \infty} s\left(t_{m}\right)=$ $1 / \alpha$. By (3.17), $\lim _{t \rightarrow \infty} x\left(t_{m}\right)=\lim _{t \rightarrow \infty}\left(x\left(t_{m}\right)+s\left(t_{m}\right)\right)-s\left(t_{m}\right)=1-1 / \alpha=(\alpha-1) / \alpha$. Therefore $\bar{x}=(\alpha-1) / \alpha$. Similarly we can show that $\underline{x}=(\alpha-1) / \alpha$. This implies that $\lim _{t \rightarrow \infty} x(t)=$ $(\alpha-1) / \alpha$, a contradiction.

Since $s(t)+x(t)$ converges and $x(t)$ converges, then $s(t)$ must also converge. Hence $\lim _{t \rightarrow \infty} s(t)=1 / \alpha$ and $\lim _{t \rightarrow \infty} x(t)=(\alpha-1) / \alpha$. It follows that $E_{2}$ is globally asymptotically stable. 
4. Hopf Bifurcations at $E_{+}$Assuming $D=\Delta=1$

Now consider the stability of $E_{+}$. The characteristic equation at $E_{+}$is

$$
\begin{aligned}
\left.P(\lambda)\right|_{E_{+}}= & (-\Delta-\lambda)\left(\left(1+\alpha x_{+}(\tau)+\lambda\right)\left(D-\alpha s_{+}(\tau)+k y_{+}(\tau)+\lambda\right)+\alpha^{2} s_{+}(\tau) x_{+}(\tau)\right) \\
& +e^{-(\Delta+\lambda) \tau} k x_{+}(\tau)\left((\lambda+1)(\lambda+D)+\alpha x_{+}(\tau)(\lambda+D)-\alpha s_{+}(\tau)(\lambda+1)\right) \\
= & (-\Delta-\lambda)\left(\left(1+\alpha x_{+}(\tau)+\lambda\right) \lambda+\alpha^{2} s_{+}(\tau) x_{+}(\tau)\right) \\
& +e^{-(\Delta+\lambda) \tau} k x_{+}(\tau)\left((\lambda+1)(\lambda+D)+\alpha x_{+}(\tau)(\lambda+D)-\alpha s_{+}(\tau)(\lambda+1)\right) \\
= & (-\Delta-\lambda)\left(\left(\frac{1}{s_{+}(\tau)}+\lambda\right) \lambda+\alpha\left(1-s_{+}(\tau)\right)\right) \\
& +e^{-\lambda \tau} \Delta\left((\lambda+1)(\lambda+D)+\frac{1-s_{+}(\tau)}{s_{+}(\tau)}(\lambda+D)-\alpha s_{+}(\tau)(\lambda+1)\right) \\
= & (-\Delta-\lambda)\left(\lambda^{2}+\frac{\lambda}{s_{+}(\tau)}+\alpha\left(1-s_{+}(\tau)\right)\right) \\
& +\Delta e^{-\lambda \tau}\left(\left(\lambda+\frac{1}{s_{+}(\tau)}\right)(\lambda+D)-\alpha s_{+}(\tau)(\lambda+1)\right)=0 .
\end{aligned}
$$

By assumption $\Delta=D=1$, and so

$$
\begin{aligned}
\left.P(\lambda)\right|_{E_{+}} & =-(\lambda+1)\left(\lambda^{2}+\frac{\lambda}{s_{+}(\tau)}+\alpha\left(1-s_{+}(\tau)\right)+e^{-\lambda \tau}\left(-\lambda+\alpha s_{+}(\tau)-\frac{1}{S_{+}(\tau)}\right)\right) \\
& =-(\lambda+1)\left(\lambda^{2}+p(\tau) \lambda+\beta(\tau)+e^{-\lambda \tau}(q \lambda+c(\tau))\right)=0
\end{aligned}
$$

where

$$
p(\tau)=\frac{1}{S_{+}(\tau)}, \quad \beta(\tau)=\alpha\left(1-s_{+}(\tau)\right), \quad q=-1, \quad c(\tau)=\alpha s_{+}(\tau)-\frac{1}{S_{+}(\tau)} .
$$

The characteristic equation at $E_{+}$has one eigenvalue equal to -1 and the others are given by solutions of the equation

$$
\lambda^{2}+p(\tau) \lambda+\beta(\tau)+e^{-\lambda \tau}(q \lambda+c(\tau))=0
$$

Lemma 4.1. Assuming $k>0, \alpha>0$, and $k(1-1 / \alpha) \geqslant 1$ so that $\tau_{c}=\ln (k(1-1 / \alpha)) \geqslant 0$, then $E_{+}$ has no zero eigenvalue for $\tau \in\left(0, \tau_{c}\right)$.

Proof. Assume that $\tau \in\left(0, \tau_{c}\right)$. By the method of contradiction, suppose that there exists a zero root of (4.4). Therefore

$$
\beta(\tau)+c(\tau)=\alpha-\frac{1}{S_{+}(\tau)}=0
$$


Noting that $\tau_{c}>0$ if and only if $k(1-1 / \alpha)>1$, for any $0<\tau<\tau_{c}$,

$$
\alpha-\frac{1}{S_{+}(\tau)}=\alpha-1-\frac{\alpha}{k} e^{\tau}>\alpha-1-\alpha\left(1-\frac{1}{\alpha}\right)=0
$$

a contradiction.

Lemma 4.2. Assume $k>0, \alpha>0, k(1-1 / \alpha)>1$. Equilibrium $E_{+}$is asymptotically stable when $\tau=0$.

Proof. For $\tau=0,(4.4)$ reduces to

$$
\lambda^{2}+p(0) \lambda+\beta(0)+(q \lambda+c(0))=\lambda^{2}+\left(\frac{1}{s_{+}(0)}-1\right) \lambda+\alpha-\frac{1}{s_{+}(0)}
$$

Both coefficients are positive, since

$$
\begin{gathered}
\frac{1}{s_{+}(0)}-1=\frac{\alpha}{k}>0, \\
\alpha-\frac{1}{s_{+}(0)}=\alpha-1-\frac{\alpha}{k}=\alpha\left(1-\frac{1}{\alpha}-\frac{1}{k}\right)>0,
\end{gathered}
$$

and $k(1-1 / \alpha)>1$ implies $1-1 / \alpha>1 / k$. Therefore, all the roots of the characteristic equation have negative real parts.

Lemma 4.3. As $\tau$ is increased from 0 , a root of (4.4) with positive real part can only appear if a root with negative real part crosses the imaginary axis.

Proof. Taking $n=2$ and $g(\lambda, \tau)=p(\tau) \lambda+(q \lambda+c(\tau)) e^{-\lambda \tau}+\beta(\tau)$ in Kuang [10, Theorem 1.4, page 66] gives

$$
\limsup _{\operatorname{Re} \lambda>0,|\lambda| \rightarrow \infty}\left|\lambda^{-2} g(\lambda, \tau)\right|=0<1
$$

Therefore, no root of (4.4) with positive real part can enter from infinity as $\tau$ increases from 0 . Hence roots with positive real part can only appear by crossing the imaginary axis.

For $\tau \neq 0$, assuming $\lambda=i \omega(\omega>0)$ is a root of $\left.P(\lambda)\right|_{E_{+}}=0$,

$$
-\omega^{2}+i p(\tau) \omega+\beta(\tau)+e^{-i \omega \tau}(i q \omega+c(\tau))=0
$$

Substituting $e^{i \theta}=\cos \theta+i \sin \theta$ into (4.10) gives

$$
-\omega^{2}+\beta(\tau)+q \omega \sin (\omega \tau)+c(\tau) \cos (\omega \tau)+i(p(\tau) \omega+q \omega \cos (\omega \tau)-c(\tau) \sin (\omega \tau))=0 .
$$


Separating the real and imaginary parts, we obtain

$$
\begin{gathered}
c(\tau) \cos (\omega \tau)+q \omega \sin (\omega \tau)=\omega^{2}-\beta(\tau), \\
c(\tau) \sin (\omega \tau)-q \omega \cos (\omega \tau)=p(\tau) \omega .
\end{gathered}
$$

Solving for $\cos (\omega \tau)$ and $\sin (\omega \tau)$ gives

$$
\begin{aligned}
& \sin (\omega \tau)=\frac{c(\tau) p(\tau) \omega+q \omega\left(\omega^{2}-\beta(\tau)\right)}{c(\tau)^{2}+q^{2} \omega^{2}}, \\
& \cos (\omega \tau)=\frac{c(\tau)\left(\omega^{2}-\beta(\tau)\right)-q p(\tau) \omega^{2}}{c(\tau)^{2}+q^{2} \omega^{2}} .
\end{aligned}
$$

Noting $\sin ^{2}(\omega \tau)+\cos ^{2}(\omega \tau)=1$, squaring both sides of equations (4.13), adding, and rearranging gives

$$
\omega^{4}+\left(p^{2}(\tau)-q^{2}-2 \beta(\tau)\right) \omega^{2}+\beta^{2}(\tau)-c^{2}(\tau)=0
$$

Solving for $\omega$, we obtain two roots $\omega_{1}(\tau)$ and $\omega_{2}(\tau)$ :

$$
\begin{aligned}
& \omega_{1}(\tau)=\frac{1}{\sqrt{2}}\left(q^{2}-p^{2}(\tau)+2 \beta(\tau)+\sqrt{\left(q^{2}-p^{2}(\tau)+2 \beta(\tau)\right)^{2}-4\left(\beta^{2}(\tau)-c^{2}(\tau)\right)}\right)^{1 / 2} \\
& =\frac{1}{S_{+}(\tau) \sqrt{2}}\left(\left(1-s_{+}(\tau)\right)\left(2 \alpha s_{+}^{2}(\tau)-s_{+}(\tau)-1\right)\right. \\
& \left.+\sqrt{\left(s_{+}^{2}(\tau)-1\right)^{2}+4 \alpha s_{+}^{2}(\tau)\left(s_{+}^{2}(\tau)-1\right)\left(1-s_{+}(\tau)\right)+4 s_{+}^{2}(\tau)\left(\alpha s_{+}^{2}(\tau)-1\right)^{2}}\right)^{1 / 2} \\
& \omega_{2}(\tau)=\frac{1}{\sqrt{2}}\left(q^{2}-p^{2}(\tau)+2 \beta(\tau)-\sqrt{\left(q^{2}-p^{2}(\tau)+2 \beta(\tau)\right)^{2}-4\left(\beta^{2}(\tau)-c^{2}(\tau)\right)}\right)^{1 / 2} \\
& =\frac{1}{S_{+}(\tau) \sqrt{2}}\left(\left(1-s_{+}(\tau)\right)\left(2 \alpha s_{+}^{2}(\tau)-s_{+}(\tau)-1\right)\right. \\
& \left.-\sqrt{\left(s_{+}^{2}(\tau)-1\right)^{2}+4 \alpha s_{+}^{2}(\tau)\left(s_{+}^{2}(\tau)-1\right)\left(1-s_{+}(\tau)\right)+4 s_{+}^{2}(\tau)\left(\alpha s_{+}^{2}(\tau)-1\right)^{2}}\right)^{1 / 2} .
\end{aligned}
$$


Define conditions $\left(H_{1}\right)$ and $\left(H_{2}\right)$ as follows:

$$
\begin{gathered}
q^{2}-p^{2}(\tau)+2 \beta(\tau)>0, \quad \beta^{2}(\tau)-c^{2}(\tau)>0, \\
\left(q^{2}-p^{2}(\tau)+2 \beta(\tau)\right)^{2}-4\left(\beta^{2}(\tau)-c^{2}(\tau)\right) \geqslant 0 \\
\left(H_{2}\right) \quad \beta^{2}(\tau)-c^{2}(\tau)<0, \quad \text { or } \quad \beta^{2}(\tau)-c^{2}(\tau)=0 \quad \text { and } \quad q^{2}-p^{2}(\tau)+2 \beta(\tau)>0 .
\end{gathered}
$$

Lemma 4.4. If $\left(H_{1}\right)$ holds for all $\tau$ in some interval $I$, then (4.14) has two positive roots $\omega_{1}(\tau) \geqslant$ $\omega_{2}(\tau)$ for all $\tau \in I$ with $\omega_{1}(\tau)>\omega_{2}(\tau)$ when all the inequalities in $\left(H_{1}\right)$ are strict. If $\left(H_{2}\right)$ holds for all $\tau$ in some interval $I$, then (4.14) has only one positive root, $\omega_{1}(\tau)$ for all $\tau \in I$. If no interval exists where either $\left(H_{1}\right)$ or $\left(H_{2}\right)$ holds, then there are no positive real roots of $(4.14)$.

Define the interval

$$
J=\left[\ln \left(\frac{k}{\alpha}\left(\frac{1}{1 / 4+\sqrt{1 / 16+1 /(2 \alpha)}}-1\right)\right), \ln \left(\frac{k(\sqrt[4]{\alpha}-1)}{\alpha}\right)\right]
$$

When the end points of $J$ are real and $J \neq \emptyset$, define

$$
I_{1}=\left[0, \tau_{c}\right) \cap J
$$

We prove that $\left(H_{1}\right)$ holds for any $\tau \in I_{1}$.

From $D=\Delta=1$,

$$
\tau_{c}=\frac{1}{\Delta} \ln \left(\frac{k}{\Delta}\left(\frac{1}{D}-\frac{1}{\alpha}\right)\right)=\ln \left(\frac{k(\alpha-1)}{\alpha}\right)
$$

If $\alpha>1$, then $\alpha>\sqrt[4]{\alpha}$. It follows that

$$
\tau_{c}>\ln \left(\frac{k(\sqrt[4]{\alpha}-1)}{\alpha}\right)
$$

Therefore,

$$
I_{1}=\left[\max \left\{0, \ln \left(\frac{k}{\alpha}\left(\frac{1}{1 / 4+\sqrt{1 / 16+1 /(2 \alpha)}}-1\right)\right)\right\}, \ln \left(\frac{k(\sqrt[4]{\alpha}-1)}{\alpha}\right)\right]
$$

Theorem 4.5. Assume $\alpha>(7+3 \sqrt{5}) / 2$ and $k>\alpha /(\sqrt[4]{\alpha}-1)$, then $I_{1}$ is not empty, and for any $\tau \in I_{1}$, but $\tau \neq \ln ((k / \alpha)(1 /(1 / 4+\sqrt{1 / 16+1 /(2 \alpha)})-1))$, condition $\left(H_{1}\right)$ holds and $\omega_{1}(\tau)>\omega_{2}(\tau)>0$. If $\tau=\ln ((k / \alpha)(1 /(1 / 4+\sqrt{1 / 16+1 /(2 \alpha)})-1)) \in I_{1}$, then $\omega_{1}(\tau)>\omega_{2}(\tau)=0$. 
Proof. For any $\alpha>(7+3 \sqrt{5}) / 2$, we have $1-1 / \sqrt[4]{\alpha}>0$, and therefore

$$
\frac{1}{\sqrt[4]{\alpha}}+\frac{1}{2}-\frac{\sqrt{5}}{2}<\frac{1}{\sqrt[4]{(7+3 \sqrt{5}) / 2}}+\frac{1}{2}-\frac{\sqrt{5}}{2}=0
$$

Hence,

$$
\begin{aligned}
& \left(\frac{1}{\sqrt[4]{\alpha}}-\frac{1}{4}\right)^{2}-\left(\sqrt{\frac{1}{16}+\frac{1}{2 \alpha}}\right)^{2}=\frac{1}{\sqrt{\alpha}}-\frac{1}{2 \sqrt[4]{\alpha}}-\frac{1}{2 \alpha} \\
& =\frac{-1}{2 \sqrt[4]{\alpha}}\left(\left(\frac{1}{\sqrt[4]{\alpha}}\right)^{3}+1-\frac{2}{\sqrt[4]{\alpha}}\right) \\
& =\frac{1}{2 \sqrt[4]{\alpha}}\left(1-\frac{1}{\sqrt[4]{\alpha}}\right)\left(\left(\frac{1}{\sqrt[4]{\alpha}}\right)^{2}+\frac{1}{\sqrt[4]{\alpha}}-1\right) \\
& =\frac{1}{2 \sqrt[4]{\alpha}}\left(1-\frac{1}{\sqrt[4]{\alpha}}\right)\left(\left(\frac{1}{\sqrt[4]{\alpha}}+\frac{1}{2}\right)^{2}-\frac{5}{4}\right) \\
& =\frac{1}{2 \sqrt[4]{\alpha}}\left(1-\frac{1}{\sqrt[4]{\alpha}}\right)\left(\frac{1}{\sqrt[4]{\alpha}}+\frac{1}{2}-\frac{\sqrt{5}}{2}\right)\left(\frac{1}{\sqrt[4]{\alpha}}+\frac{1}{2}+\frac{\sqrt{5}}{2}\right)<0 .
\end{aligned}
$$

Therefore, $1 / \sqrt[4]{\alpha}-1 / 4<\sqrt{1 / 16+1 /(2 \alpha)}$. Since $1 / 4+\sqrt{1 / 16+1 /(2 \alpha)}<1 / 4+$ $\sqrt{1 / 16+1 /(7+3 \sqrt{5})}<1$, it follows that

$$
\frac{1}{\sqrt[4]{\alpha}}<\frac{1}{4}+\sqrt{\frac{1}{16}+\frac{1}{2 \alpha}}<1
$$

Hence,

$$
\ln \left(\frac{k}{\alpha}\left(\frac{1}{1 / 4+\sqrt{1 / 16+1 /(2 \alpha)}}-1\right)\right)<\ln \left(\frac{k(\sqrt[4]{\alpha}-1)}{\alpha}\right)
$$

From $k>\alpha /(\sqrt[4]{\alpha}-1)$, we have $\ln (k(\sqrt[4]{\alpha}-1) / \alpha)>0$. Therefore,

$$
\max \left\{0, \ln \left(\frac{k}{\alpha}\left(\frac{1}{1 / 4+\sqrt{1 / 16+1 /(2 \alpha)}}-1\right)\right)\right\}<\ln \left(\frac{k(\sqrt[4]{\alpha}-1)}{\alpha}\right)
$$

and so $I_{1}$ is not empty. Noting $s_{+}(\tau)=1 /\left(1+(\alpha \Delta / k) e^{\Delta \tau}\right)$ and $\Delta=1$, for any $\tau \in I_{1}$, but $\tau \neq \ln ((k / \alpha)(1 /(1 / 4+\sqrt{1 / 16+1 /(2 \alpha)})-1))$, we have $s_{+}(\tau) \in[1 / \sqrt[4]{\alpha}, 1 / 4+\sqrt{1 / 16+1 /(2 \alpha)})$. 
International Journal of Differential Equations

In what follows, we intend to show that for any such $\tau$, condition $\left(H_{1}\right)$ holds. From (4.3),

$$
\begin{aligned}
q^{2}-p^{2}(\tau)+2 \beta(\tau) & =(-1)^{2}-\frac{1}{s_{+}^{2}(\tau)}+2 \alpha\left(1-s_{+}(\tau)\right) \\
& =\frac{\left(1-s_{+}(\tau)\right) 2 \alpha}{s_{+}^{2}(\tau)}\left(s_{+}(\tau)^{2}-\frac{s_{+}(\tau)}{2 \alpha}-\frac{1}{2 \alpha}\right) \\
& =\frac{\left(1-s_{+}(\tau)\right) 2 \alpha}{s_{+}^{2}(\tau)}\left(\left(s_{+}(\tau)-\frac{1}{4 \alpha}\right)^{2}-\frac{1}{16 \alpha^{2}}-\frac{1}{2 \alpha}\right)
\end{aligned}
$$

Since $s_{+}(\tau)<1$, to show that the first inequality in $\left(H_{1}\right)$ holds, it suffices to show that the factor on the right-hand side of the above expression is positive. Since $\alpha>(7+3 \sqrt{5}) / 2$, $1 / \sqrt{\alpha}-1 /(4 \alpha)=(1 / \sqrt{\alpha})(1-1 /(4 \sqrt{\alpha}))>0$, and

$$
\begin{aligned}
\left(\sqrt{\frac{1}{16 \alpha^{2}}+\frac{1}{2 \alpha}}\right)^{2}-\left(\frac{1}{\sqrt{\alpha}}-\frac{1}{4 \alpha}\right)^{2} & =\frac{1}{16 \alpha^{2}}+\frac{1}{2 \alpha}-\frac{1}{\alpha}+\frac{1}{2 \alpha \sqrt{\alpha}}-\frac{1}{16 \alpha^{2}} \\
& =\frac{1}{2 \alpha}\left(\frac{1}{\sqrt{\alpha}}-1\right)<0
\end{aligned}
$$

Since $1 / \sqrt{\alpha}<1 / \sqrt[4]{\alpha}$ for $\alpha>(7+3 \sqrt{5}) / 2$,

$$
\frac{1}{4 \alpha}+\sqrt{\frac{1}{16 \alpha^{2}}+\frac{1}{2 \alpha}}<\frac{1}{\sqrt{\alpha}}<\frac{1}{\sqrt[4]{\alpha}} .
$$

For any $s_{+}(\tau)>1 / \sqrt[4]{\alpha}$,

$$
S_{+}(\tau)-\frac{1}{4 \alpha} \geqslant \frac{1}{\sqrt[4]{\alpha}}-\frac{1}{4 \alpha}>\sqrt{\frac{1}{16 \alpha^{2}}+\frac{1}{2 \alpha}}
$$

Hence,

$$
\left(s_{+}(\tau)-\frac{1}{4 \alpha}\right)^{2} \geqslant \frac{1}{16 \alpha^{2}}+\frac{1}{2 \alpha} .
$$


Next consider the second inequality in $\left(H_{1}\right)$. For $\alpha>(7+3 \sqrt{5}) / 2$, since $1 / \sqrt[4]{\alpha}>1 / \alpha, s_{+}(\tau) \geqslant$ $1 / \sqrt[4]{\alpha}>1 / \alpha$. Therefore, $\alpha S_{+}(\tau)>1$. For $s_{+}(\tau) \in[1 / \sqrt[4]{\alpha}, 1 / 4+\sqrt{1 / 16+1 /(2 \alpha)})$

$$
\begin{aligned}
\beta^{2}(\tau)-c^{2}(\tau) & =(\beta(\tau)-c(\tau))(\beta(\tau)+c(\tau)) \\
& =\left(\alpha-2 \alpha s_{+}(\tau)+\frac{1}{s_{+}(\tau)}\right)\left(\alpha-\frac{1}{s_{+}(\tau)}\right) \\
& =-\frac{2 \alpha}{s_{+}^{2}(\tau)}\left(s_{+}^{2}(\tau)-\frac{s_{+}(\tau)}{2}-\frac{1}{2 \alpha}\right)\left(\alpha s_{+}(\tau)-1\right) \\
& =-\frac{2 \alpha}{s_{+}^{2}(\tau)}\left(\left(s_{+}(\tau)-\frac{1}{4}\right)^{2}-\frac{1}{16}-\frac{1}{2 \alpha}\right)\left(\alpha s_{+}(\tau)-1\right)>0
\end{aligned}
$$

Finally,

$$
\begin{aligned}
\left(q^{2}-\right. & \left.p^{2}(\tau)+2 \beta(\tau)\right)^{2}-4\left(\beta^{2}(\tau)-c^{2}(\tau)\right)=\left(q^{2}-p^{2}(\tau)\right)\left(q^{2}-p^{2}(\tau)+4 \beta(\tau)\right)+4 c^{2}(\tau) \\
& =\left(1-\frac{1}{s_{+}^{2}(\tau)}\right)\left(1-\frac{1}{s_{+}^{2}(\tau)}+4 \alpha\left(1-s_{+}(\tau)\right)\right)+4\left(\alpha s_{+}(\tau)-\frac{1}{s_{+}(\tau)}\right)^{2} \\
& =\left(1-\frac{1}{s_{+}^{2}(\tau)}\right)^{2}+4 \alpha\left(1-\frac{1}{s_{+}^{2}(\tau)}\right)\left(1-s_{+}(\tau)\right)+4 s_{+}(\tau)^{2} \alpha^{2}-8 \alpha+\frac{4}{s_{+}^{2}(\tau)} \\
& =4 s_{+}(\tau)^{2} \alpha^{2}+4 \alpha\left(\left(1-\frac{1}{s_{+}^{2}(\tau)}\right)\left(1-s_{+}(\tau)\right)\right)-8 \alpha+\left(1-\frac{1}{s_{+}^{2}(\tau)}\right)^{2}+\frac{4}{s_{+}^{2}(\tau)} \\
& =4 s_{+}(\tau)^{2} \alpha^{2}+4 \alpha\left(\left(1-\frac{1}{s_{+}^{2}(\tau)}\right)\left(1-s_{+}(\tau)\right)-2\right)+\left(1+\frac{1}{s_{+}^{2}(\tau)}\right)^{2} \\
& =\left(\alpha-\alpha_{1}\right)\left(\alpha-\alpha_{2}\right),
\end{aligned}
$$

where

$$
\begin{aligned}
& \alpha_{1}=\frac{2-\left(1-1 / s_{+}^{2}(\tau)\right)\left(1-s_{+}(\tau)\right)+\sqrt{\left(1 / s_{+}^{2}(\tau)+2 s_{+}(\tau)+1\right)\left(1 / s_{+}(\tau)-1\right)^{2}}}{2 s_{+}^{2}(\tau)}, \\
& \alpha_{2}=\frac{2-\left(1-1 / s_{+}^{2}(\tau)\right)\left(1-s_{+}(\tau)\right)-\sqrt{\left(1 / s_{+}^{2}(\tau)+2 s_{+}(\tau)+1\right)\left(1 / s_{+}(\tau)-1\right)^{2}}}{2 s_{+}^{2}(\tau)} .
\end{aligned}
$$


Since $s_{+}(\tau)<1$,

$$
\begin{gathered}
2-\left(1-\frac{1}{s_{+}^{2}(\tau)}\right)\left(1-s_{+}(\tau)\right)=s_{+}(\tau)+1+\frac{1-s_{+}(\tau)}{s_{+}^{2}(\tau)}>0 \\
\left(2-\left(1-\frac{1}{s_{+}^{2}(\tau)}\right)\left(1-s_{+}(\tau)\right)\right)^{2} \\
>\left(2-\left(1-\frac{1}{s_{+}^{2}(\tau)}\right)\left(1-s_{+}(\tau)\right)\right)^{2}-s_{+}^{2}(\tau)\left(1+\frac{1}{s_{+}^{2}(\tau)}\right)^{2} \\
=\left(\frac{1}{s_{+}^{2}(\tau)}+2 s_{+}(\tau)+1\right)\left(\frac{1}{s_{+}(\tau)}-1\right)^{2}>0 .
\end{gathered}
$$

It follows that $0<\alpha_{2}<\alpha_{1}$. Again noting that $s_{+}(\tau)<1$,

$$
\begin{aligned}
\alpha_{1} & <\frac{2-\left(1-1 / s_{+}^{2}(\tau)\right)\left(1-s_{+}(\tau)\right)+\sqrt{\left(1 / s_{+}^{2}(\tau)+2 / s_{+}(\tau)+1\right)\left(1 / s_{+}(\tau)-1\right)^{2}}}{2 s_{+}^{2}(\tau)} \\
& =\frac{2-\left(1-1 / s_{+}^{2}(\tau)\right)\left(1-s_{+}(\tau)\right)+\left(1 / s_{+}(\tau)+1\right)\left(1 / s_{+}(\tau)-1\right)}{2 s_{+}^{2}(\tau)} \\
& =\frac{2-\left(1-s_{+}(\tau)-1 / s_{+}^{2}(\tau)+1 / s_{+}(\tau)\right)+1 / s_{+}^{2}(\tau)-1}{2 s_{+}^{2}(\tau)} \\
& =\frac{s_{+}(\tau)+2 / s_{+}^{2}(\tau)-1 / s_{+}(\tau)}{2 s_{+}^{2}(\tau)}=\frac{1}{2}\left(\frac{2}{s_{+}^{4}(\tau)}-\left(\frac{1}{s_{+}^{3}(\tau)}-\frac{1}{s_{+}(\tau)}\right)\right) \\
& <\frac{1}{2} \frac{2}{s_{+}^{4}(\tau)}=\frac{1}{s_{+}^{4}(\tau)} .
\end{aligned}
$$

Hence, for any $s_{+}(\tau)>1 / \sqrt[4]{\alpha}$, we have $\alpha>1 / s_{+}^{4}(\tau)>\alpha_{1}>\alpha_{2}$. This leads to

$$
\left(q^{2}-p^{2}(\tau)+2 \beta(\tau)\right)^{2}-4\left(\beta^{2}(\tau)-c^{2}(\tau)\right)=\left(\alpha-\alpha_{1}\right)\left(\alpha-\alpha_{2}\right)>0
$$

Therefore, $\left(H_{1}\right)$ holds for any $\tau \in I_{1}$. By Lemma 4.4 , both $\omega_{1}(\tau)>0$ and $\omega_{2}(\tau)>0$.

If $\tau=\ln ((k / \alpha)(1 /(1 / 4+\sqrt{1 / 16+1 /(2 \alpha)})-1)) \in I_{1}$, we have $s_{+}(\tau)=1 / 4+$ $\sqrt{1 / 16+1 /(2 \alpha)}$. Noting (4.25), we obtain

$$
\begin{gathered}
q^{2}-p^{2}(\tau)+2 \beta(\tau)>0, \quad \beta^{2}(\tau)-c^{2}(\tau)=0 \\
\left(q^{2}-p^{2}(\tau)+2 \beta(\tau)\right)^{2}-4\left(\beta^{2}(\tau)-c^{2}(\tau)\right)>0
\end{gathered}
$$

By (4.15), it follows that $\omega_{1}(\tau)>0$ and $\omega_{2}(\tau)=0$. 
Now we define interval $I_{2}$ and prove that $\left(H_{2}\right)$ holds on $I_{2}$

$$
I_{2}:=\left[0, \tau_{c}\right) \cap\left(-\infty, \ln \left(\frac{k}{\alpha}\left(\frac{1}{1 / 4+\sqrt{1 / 16+1 /(2 \alpha)}}-1\right)\right)\right] .
$$

In the following theorem, we consider the case that parameters are chosen so that

$$
I_{2}=\left[0, \ln \left(\frac{k}{\alpha}\left(\frac{1}{1 / 4+\sqrt{1 / 16+1 /(2 \alpha)}}-1\right)\right)\right]
$$

Theorem 4.6. Assume $\alpha>1$ and $k>\alpha(1 /(1 / 4+\sqrt{1 / 16+1 /(2 \alpha)})-1)^{-1}$. Interval $I_{2}$ given by (4.41) is not empty. For any $\tau \in I_{2},\left(H_{2}\right)$ holds and hence $\omega_{1}(\tau)>0$.

Proof. Assume $\alpha>1$. Letting

$$
G(\alpha)=\frac{1}{4}+\sqrt{\frac{1}{16}+\frac{1}{2 \alpha}}-\frac{1}{\alpha}
$$

then

$$
\frac{\mathrm{d}}{\mathrm{d} \alpha} G(\alpha)=-\frac{1}{4 \alpha^{2} \sqrt{1 / 16+1 /(2 \alpha)}}+\frac{1}{\alpha^{2}}=\frac{1}{\alpha^{2}}\left(\frac{-1}{\sqrt{1+8 / \alpha}}+1\right)>0
$$

$G(\alpha)$ is an increasing function of $\alpha$ and $G(1)=0 . G(\alpha)>G(1)$ implies that $1 / 4+$ $\sqrt{1 / 16+1 /(2 \alpha)}-1 / \alpha>0$. Therefore

$$
1>\frac{1}{4}+\sqrt{\frac{1}{16}+\frac{1}{2 \alpha}}>\frac{1}{\alpha} .
$$

This gives

$$
\alpha-1>\frac{1}{1 / 4+\sqrt{1 / 16+1 /(2 \alpha)}}-1>0 .
$$

By assumption $k>\alpha(1 /(1 / 4+\sqrt{1 / 16+1 /(2 \alpha)})-1)^{-1}$, we obtain

$$
\frac{k(\alpha-1)}{\alpha}>\frac{k}{\alpha}\left(\frac{1}{1 / 4+\sqrt{1 / 16+1 /(2 \alpha)}}-1\right)>1 .
$$

Noting that $D=\Delta=1$ and recalling the definition of $\tau_{c}$ given in (3.2),

$$
\tau_{c}=\ln \left(\frac{k(\alpha-1)}{\alpha}\right)>\ln \left(\frac{k}{\alpha}\left(\frac{1}{1 / 4+\sqrt{1 / 16+1 /(2 \alpha)}}-1\right)\right)>0 .
$$


Therefore, $I_{2}$ given by (4.41) is not empty. For any $\tau \in I_{2}$, noting $s_{+}(\tau)=1 /\left(1+(\alpha \Delta / k) e^{\Delta \tau}\right)$ and $\Delta=1$, we have $s_{+}(\tau) \in[1 / 4+\sqrt{1 / 16+1 /(2 \alpha)}, 1 /(1+\alpha) / k) \subset[1 / 4+\sqrt{1 / 16+1 /(2 \alpha)}, 1)$. In what follows, we intend to show for any $\tau \in I_{2}$, or equivalently $s_{+}(\tau) \in[1 / 4+$ $\sqrt{1 / 16+1 /(2 \alpha)}, 1),\left(H_{2}\right)$ holds. For any $s_{+}(\tau) \in[1 / 4+\sqrt{1 / 16+1 /(2 \alpha)}, 1)$, by $(4.44)$, it follows that $s_{+}(\tau)>1 / \alpha$ and so $\alpha s_{+}(\tau)>1$. Hence,

$$
\beta^{2}(\tau)-c^{2}(\tau)=-\frac{2 \alpha}{s_{+}^{2}(\tau)}\left(\left(s_{+}(\tau)-\frac{1}{4}\right)^{2}-\frac{1}{16}-\frac{1}{2 \alpha}\right)\left(\alpha S_{+}(\tau)-1\right) \leqslant 0
$$

For any $s_{+}(\tau) \in[1 / 4+\sqrt{1 / 16+1 /(2 \alpha)}, 1)$, we have $s_{+}(\tau)>1 /(4 \alpha)+\sqrt{1 /\left(16 \alpha^{2}\right)+1 /(2 \alpha)}$, since $1 / 4>1 /(4 \alpha)$ and $1 / 16>1 /\left(16 \alpha^{2}\right)$ imply that

$$
\frac{1}{4}+\sqrt{\frac{1}{16}+\frac{1}{2 \alpha}}>\frac{1}{4 \alpha}+\sqrt{\frac{1}{16 \alpha^{2}}+\frac{1}{2 \alpha}} .
$$

Therefore,

$$
q^{2}-p^{2}(\tau)+2 \beta(\tau)=\frac{\left(1-s_{+}(\tau)\right) 2 \alpha}{s_{+}^{2}(\tau)}\left(\left(s_{+}(\tau)-\frac{1}{4 \alpha}\right)^{2}-\frac{1}{16 \alpha^{2}}-\frac{1}{2 \alpha}\right)>0 .
$$

Condition $\left(H_{2}\right)$ holds. By Lemma 4.4, $\omega_{1}(\tau)>0$. consider

Next, to determine whether (4.2) has a pair of pure imaginary eigenvalues, we

$$
\begin{aligned}
& c^{2}(\tau)+q^{2} \omega^{2} \\
& =\left(\alpha S_{+}(\tau)-\frac{1}{S_{+}(\tau)}\right)^{2}+(-1)^{2} \omega^{2}=\left(\alpha s_{+}(\tau)-\frac{1}{S_{+}(\tau)}\right)^{2}+\omega^{2}, \\
& c(\tau)(p(\tau) \omega)+q \omega\left(\omega^{2}-\beta(\tau)\right) \\
& =\omega\left(c(\tau) p(\tau)+q\left(\omega^{2}-\beta(\tau)\right)\right) \\
& =\omega\left(\alpha-\frac{1}{s_{+}^{2}(\tau)}-\omega^{2}+\alpha\left(1-s_{+}(\tau)\right)\right), \\
& c(\tau)\left(\omega^{2}-\beta(\tau)\right)+q \omega(-p(\tau) \omega) \\
& =\left(\alpha S_{+}(\tau)-\frac{1}{S_{+}(\tau)}\right)\left(\omega^{2}-\alpha\left(1-s_{+}(\tau)\right)\right)+(-1) \omega\left(\frac{-\omega}{s_{+}(\tau)}\right) \\
& =\alpha\left(s_{+}(\tau) \omega^{2}-\left(1-s_{+}(\tau)\right)\left(\alpha S_{+}(\tau)-\frac{1}{s_{+}(\tau)}\right)\right) .
\end{aligned}
$$


We obtain

$$
\begin{aligned}
& \sin (\omega \tau)=-\omega \frac{\omega^{2}-\left(\alpha-1 / s_{+}^{2}(\tau)\right)-\alpha\left(1-s_{+}(\tau)\right)}{\left(\alpha s_{+}(\tau)-1 / s_{+}(\tau)\right)^{2}+\omega^{2}}, \\
& \cos (\omega \tau)=\alpha s_{+}(\tau) \frac{\omega^{2}-\left(1-s_{+}(\tau)\right)\left(\alpha-1 / s_{+}^{2}(\tau)\right)}{\left(\alpha s_{+}(\tau)-1 / s_{+}(\tau)\right)^{2}+\omega^{2}} .
\end{aligned}
$$

If there exists $(\tau, \omega)$ satisfying (4.52), then (4.2) has a pair of pure imaginary roots $\pm i \omega$. A necessary condition for (4.52) to have solutions is $\alpha s_{+}^{2}(\tau) \neq 1$. Otherwise, $\alpha s_{+}^{2}(\tau)=1$, and the second equation of (4.52) becomes $\cos (\omega \tau)=\alpha s_{+}(\tau)$. However, for any $\tau \in\left(0, \tau_{c}\right)$, we have $\alpha s_{+}(\tau)>1$, since

$$
\alpha S_{+}(\tau)=\frac{\alpha}{1+\alpha e^{\tau} / k}>\frac{\alpha}{1+(\alpha / k) k(1-1 / \alpha)}=\frac{\alpha}{1+\alpha(1-1 / \alpha)}=\frac{\alpha}{\alpha}=1
$$

Hence, the second equation of (4.52) has no solution. Assume $\alpha s_{+}^{2}(\tau) \neq 1$ for $\omega \geqslant 0$ and $\tau \in$ $\left[0, \tau_{c}\right]$ and denote the right-hand sides of $(4.52)$ by

$$
\begin{aligned}
h_{1}(\omega, \tau) & =\frac{c(\tau)(p(\tau) \omega)+q \omega\left(\omega^{2}-\beta(\tau)\right)}{c^{2}(\tau)+q^{2} \omega^{2}} \\
& =-\omega \frac{\omega^{2}-\left(\alpha-1 / s_{+}^{2}(\tau)\right)-\alpha\left(1-s_{+}(\tau)\right)}{\left(\alpha s_{+}(\tau)-1 / s_{+}(\tau)\right)^{2}+\omega^{2}}, \\
h_{2}(\omega, \tau) & =\frac{c(\tau)\left(\omega^{2}-\beta(\tau)\right)+q \omega(-p(\tau) \omega)}{c^{2}(\tau)+q^{2} \omega^{2}} \\
& =\alpha s_{+}(\tau) \frac{\omega^{2}-\left(1-s_{+}(\tau)\right)\left(\alpha-1 / s_{+}^{2}(\tau)\right)}{\left(\alpha s_{+}(\tau)-1 / s_{+}(\tau)\right)^{2}+\omega^{2}} .
\end{aligned}
$$

Define functions

$$
\begin{aligned}
& \theta_{1}(\tau)=\arccos \left(h_{2}\left(\omega_{1}(\tau), \tau\right)\right) \quad \text { if } h_{2}\left(\omega_{1}(\tau), \tau\right) \in[-1,1] \\
& \theta_{2}(\tau)=\arccos \left(h_{2}\left(\omega_{2}(\tau), \tau\right)\right) \quad \text { if } h_{2}\left(\omega_{2}(\tau), \tau\right) \in[-1,1]
\end{aligned}
$$


Lemma 4.7. Assume $\alpha>(7+3 \sqrt{5}) / 2$ and $k>\alpha /(\sqrt[4]{\alpha}-1)$. For any $\tau \in I_{1}$ given by (4.19), there exists $\epsilon_{j}>0$ and $\theta_{j}(\tau)$ with $\epsilon_{j} \leqslant \theta_{j}(\tau) \leqslant \pi(j=1,2)$ such that

$$
\begin{aligned}
& \sin \left(\theta_{j}(\tau)+2 n \pi\right)=h_{1}\left(\omega_{j}(\tau), \tau\right), \\
& \cos \left(\theta_{j}(\tau)+2 n \pi\right)=h_{2}\left(\omega_{j}(\tau), \tau\right) .
\end{aligned} \quad n=0,1,2, \ldots,
$$

Proof. For any $\tau \in I_{1}$, by Theorem $4.5, \omega_{1}(\tau)>0$ and $\omega_{2}(\tau) \geqslant 0$. It is easy to see that $h_{1}(0, \tau)=$ 0 and $\lim _{\omega \rightarrow+\infty} h_{1}(\omega, \tau)=-\infty$. There are two roots of $h_{1}(z, \tau)=0, z_{1}=0$ and

$$
z_{2}(\tau)=\sqrt{\alpha-\frac{1}{s_{+}^{2}(\tau)}+\alpha\left(1-s_{+}(\tau)\right)}
$$

Hence, $h_{1}(\omega, \tau)>0$ for $0<\omega<z_{2}(\tau)$. For any $\tau \in I_{1}$, as shown in Theorem 4.5, $s_{+}(\tau) \in$ $[1 / \sqrt[4]{\alpha}, 1 / 4+\sqrt{1 / 16+1 /(2 \alpha)}]$. This implies $s_{+}(\tau) \geqslant 1 / \sqrt[4]{\alpha}>1 / \sqrt{\alpha}>1 / \alpha$. Therefore, $\alpha s_{+}^{2}(\tau)>$ 1 and $\alpha s_{+}(\tau)>1$. The function $h_{2}(\omega, \tau)$ is monotonically increasing for $\omega \geqslant 0$, since

$$
\begin{aligned}
\frac{\partial h_{2}(\omega, \tau)}{\partial \omega} & =\alpha s_{+}(\tau) \frac{2 \omega\left(\left(\alpha s_{+}(\tau)-1 / s_{+}(\tau)\right)^{2}+\omega^{2}\right)-2 \omega\left(\omega^{2}-\left(1-s_{+}(\tau)\right)\left(\alpha-1 / s_{+}^{2}(\tau)\right)\right)}{\left(\left(\alpha s_{+}(\tau)-1 / s_{+}(\tau)\right)^{2}+\omega^{2}\right)^{2}} \\
& =2 \alpha s_{+}(\tau) \omega \frac{\left(\alpha s_{+}(\tau)-1 / s_{+}(\tau)\right)^{2}+\left(1-s_{+}(\tau)\right)\left(\alpha-1 / s_{+}^{2}(\tau)\right)}{\left(\left(\alpha s_{+}(\tau)-1 / s_{+}(\tau)\right)^{2}+\omega^{2}\right)^{2}} \\
& =2 \alpha s_{+}(\tau) \omega \frac{\left(\alpha-1 / s_{+}^{2}(\tau)\right)\left(s_{+}^{2}(\tau)\left(\alpha-1 / s_{+}^{2}(\tau)\right)+1-s_{+}(\tau)\right)}{\left(\left(\alpha s_{+}(\tau)-1 / s_{+}(\tau)\right)^{2}+\omega^{2}\right)^{2}} \\
& =2 \alpha s_{+}(\tau) \omega \frac{\left(\alpha-1 / s_{+}^{2}(\tau)\right) s_{+}(\tau)\left(\alpha s_{+}(\tau)-1\right)}{\left(\left(\alpha s_{+}(\tau)-1 / s_{+}(\tau)\right)^{2}+\omega^{2}\right)^{2}} \\
& =2 \alpha \omega \frac{\left(\alpha s_{+}^{2}(\tau)-1\right)\left(\alpha s_{+}(\tau)-1\right)}{\left(\left(\alpha s_{+}(\tau)-1 / s_{+}(\tau)\right)^{2}+\omega^{2}\right)^{2}} \geqslant 0 .
\end{aligned}
$$

Since $s_{+}(\tau)<1$,

$$
h_{2}(0, \tau)=-\alpha s_{+}(\tau) \frac{\left(1-s_{+}(\tau)\right)\left(\alpha-1 / s_{+}^{2}(\tau)\right)}{\left(\alpha s_{+}(\tau)-1 / s_{+}(\tau)\right)^{2}}=-\frac{\alpha s_{+}(\tau)\left(1-s_{+}(\tau)\right)}{\alpha s_{+}^{2}(\tau)-1}<0 .
$$


Also, $\lim _{\omega \rightarrow \infty} h_{2}(\omega, \tau)=\alpha s_{+}(\tau)>1$. Therefore, there exists a unique $\omega=l_{\max }(\tau)=$ $\sqrt{\alpha-1 / s_{+}^{2}(\tau)}>0$, such that $h_{2}\left(l_{\max }(\tau), \tau\right)=1$. Solving $h_{2}\left(l_{\max }, \tau\right)=1$ for $l_{\max }$ and noting that $\alpha-1 / s_{+}^{2}(\tau) \neq 0$, it is easy to see that $l_{\max }(\tau)=\sqrt{\alpha-1 / s_{+}^{2}(\tau)}$

$$
\begin{aligned}
l_{\max }^{2}\left(\alpha s_{+}(\tau)-1\right) & =\alpha s_{+}(\tau)\left(1-s_{+}(\tau)\right)\left(\alpha-\frac{1}{s_{+}^{2}(\tau)}\right)+\left(\alpha s_{+}(\tau)-\frac{1}{s_{+}(\tau)}\right)^{2} \\
& =\alpha s_{+}(\tau)\left(\alpha-\frac{1}{s_{+}^{2}(\tau)}-\alpha s_{+}(\tau)+\frac{1}{S_{+}(\tau)}\right)+\alpha^{2} s_{+}^{2}(\tau)-2 \alpha+\frac{1}{s_{+}^{2}(\tau)} \\
& =\alpha^{2} s_{+}(\tau)-\frac{\alpha}{s_{+}(\tau)}-\alpha^{2} s_{+}^{2}(\tau)+\alpha+\alpha^{2} s_{+}^{2}(\tau)-2 \alpha+\frac{1}{s_{+}^{2}(\tau)} \\
& =\alpha^{2} s_{+}(\tau)-\frac{\alpha}{s_{+}(\tau)}-\alpha+\frac{1}{s_{+}^{2}(\tau)} \\
& =\left(\alpha-\frac{1}{s_{+}^{2}(\tau)}\right)\left(\alpha s_{+}(\tau)-1\right) .
\end{aligned}
$$

Then, $h_{2}(\omega, \tau) \leqslant 1$ for any $\omega \in\left[0, l_{\max }(\tau)\right]$. Since $s_{+}(\tau)<1, l_{\max }(\tau)<z_{2}(\tau)$. Therefore, $h_{1}(\omega, \tau)>0$ for any $\omega \in\left[0, l_{\max }(\tau)\right]$. Since $\omega_{1}(\tau)$ is a positive root of $h_{1}^{2}(\omega, \tau)+h_{2}^{2}(\omega, \tau)=$ 1 , we have $h_{2}\left(\omega_{1}(\tau), \tau\right) \leqslant 1$, which implies that $0<\omega_{1}(\tau) \leqslant l_{\max }(\tau)<z_{2}(\tau)$. Therefore, $h_{1}\left(\omega_{1}(\tau), \tau\right)>0$, and so $h_{1}\left(\omega_{1}(\tau), \tau\right)=\sqrt{1-h_{2}\left(\omega_{1}(\tau), \tau\right)}$. In fact,

$$
\omega_{1}(\tau)<l_{\max }(\tau)
$$

since

$$
h_{2}^{2}\left(l_{\max }(\tau), \tau\right)+h_{1}^{2}\left(l_{\max }(\tau), \tau\right)=1+h_{1}^{2}\left(l_{\max }(\tau), \tau\right)>1 .
$$

Thus, $\theta_{1}(\tau)$ is defined and $0 \leqslant \theta_{1}(\tau) \leqslant \pi$. Since $\cos \left(\theta_{1}(\tau)+2 n \pi\right)=h_{2}\left(\omega_{1}(\tau), \tau\right)$,

$$
\begin{aligned}
\sin \left(\theta_{1}(\tau)+2 n \pi\right) & =\sqrt{1-\cos ^{2}\left(\theta_{1}(\tau)+2 n \pi\right)} \\
& =\sqrt{1-h_{2}^{2}\left(\omega_{1}(\tau), \tau\right)} \\
& =h_{1}\left(\omega_{1}(\tau), \tau\right) .
\end{aligned}
$$

Hence, $\theta_{1}(\tau)$ satisfies (4.56). From (4.61), $h_{2}\left(\omega_{1}(\tau), \tau\right)<h_{2}\left(l_{\max }(\tau), \tau\right)=1$, and so $\theta_{1}(\tau)>0$. Since $\theta_{1}(\tau)$ is continuous on the interval $I_{1}$ and $I_{1}$ is closed, there exists $\epsilon_{1}>0$ such that $\theta_{1}(\tau) \geqslant \epsilon_{1}$. Similarly we can prove the existence of $\theta_{2}(\tau)$.

Lemma 4.8. Assume $\alpha>1$ and $k>\alpha(1 /(1 / 4+\sqrt{1 / 16+1 / 2 \alpha})-1)^{-1}$. For any $\tau \in I_{2}$ given by (4.41), there exists $\epsilon>0$ and $\theta_{1}(\tau)$ such that $\epsilon \leqslant \theta_{1}(\tau)<\pi$ and $\theta_{1}(\tau)$ satisfies (4.56) for $j=1$. 
Proof. For any $\tau \in I_{2}$, by Theorem 4.6, only $\omega_{1}(\tau)>0$.

As in Lemma 4.7, we have $h_{1}(\omega, \tau)>0$ for $0<\omega<z_{2}(\tau)$. For any $\tau \in I_{2}$, as shown in Theorem 4.6, $s_{+}(\tau) \in[1 / 4+\sqrt{1 / 16+1 /(2 \alpha)}, 1)$. Letting

$$
G(\alpha)=\frac{1}{4}+\sqrt{\frac{1}{16}+\frac{1}{2 \alpha}}-\frac{1}{\sqrt{\alpha}}, \quad \alpha>1
$$

we have

$$
\frac{\mathrm{d}}{\mathrm{d} \alpha} G(\alpha)=-\frac{1}{4 \alpha^{2} \sqrt{1 / 16+1 /(2 \alpha)}}+\frac{1}{2 \alpha \sqrt{\alpha}}=\frac{1}{2 \alpha}\left(\frac{-1}{\sqrt{\alpha^{2} / 4+2 \alpha}}+\frac{1}{\sqrt{\alpha}}\right)>0 .
$$

$G(\alpha)$ is an increasing function and $G(1)=0$. Since $G(\alpha)>G(1)$, it follows that $1 / 4+$ $\sqrt{1 / 16+1 /(2 \alpha)}>1 / \sqrt{\alpha}$. Therefore, for any $s_{+}(\tau)>1 / 4+\sqrt{1 / 16+1 /(2 \alpha)}$, we obtain $s_{+}(\tau)>1 / \sqrt{\alpha}$, or equivalently $\alpha>1 / s_{+}^{2}(\tau)$. Since

$$
\frac{\partial h_{2}(\omega, \tau)}{\partial \omega}=2 \alpha s_{+}(\tau) \omega \frac{\left(\alpha s_{+}(\tau)-1 / s_{+}(\tau)\right)^{2}+\left(1-s_{+}(\tau)\right)\left(\alpha-1 / s_{+}^{2}(\tau)\right)}{\left(\left(\alpha s_{+}(\tau)-1 / s_{+}(\tau)\right)^{2}+\omega^{2}\right)^{2}} \geqslant 0,
$$

$h_{2}(\omega, \tau)$ is monotonically increasing for any $\omega \geqslant 0$. For any $s_{+}(\tau)>1 / 4+\sqrt{1 / 16+1 /(2 \alpha)}$,

$$
\begin{aligned}
\left(s_{+}(\tau)-\frac{1}{4}\right)^{2}-\frac{1}{16}-\frac{1}{2 \alpha} & =s_{+}^{2}(\tau)-\frac{s_{+}(\tau)}{2}-\frac{1}{2 \alpha}=\frac{1}{2 \alpha}\left(2 \alpha s_{+}^{2}(\tau)-\alpha s_{+}(\tau)-1\right) \\
& =\frac{1}{2 \alpha}\left(\alpha s_{+}^{2}(\tau)-1-\left(\alpha s_{+}(\tau)-\alpha s_{+}^{2}(\tau)\right)\right) \\
& =\frac{\alpha s_{+}^{2}(\tau)-1}{2 \alpha}\left(1-\frac{\alpha s_{+}(\tau)\left(1-s_{+}(\tau)\right)}{\alpha s_{+}^{2}(\tau)-1}\right)>0
\end{aligned}
$$

which implies that $\left(\alpha s_{+}(\tau)\left(1-s_{+}(\tau)\right)\right) /\left(\alpha s_{+}^{2}(\tau)-1\right)<1$. Hence,

$$
0>h_{2}(0, \tau)=-\alpha S_{+}(\tau) \frac{\left(1-s_{+}(\tau)\right)\left(\alpha-1 / s_{+}^{2}(\tau)\right)}{\left(\alpha S_{+}(\tau)-1 / s_{+}(\tau)\right)^{2}}=-\frac{\alpha s_{+}(\tau)\left(1-s_{+}(\tau)\right)}{\alpha s_{+}^{2}(\tau)-1}>-1 .
$$

For $\tau \in\left[0, \tau_{c}\right), \lim _{\omega \rightarrow \infty} h_{2}(\omega, \tau)=\alpha s_{+}(\tau)>1$, since

$$
\alpha S_{+}(\tau)=\frac{\alpha}{1+\alpha e^{\tau} / k}>\frac{\alpha}{1+(\alpha / k) k(1-1 / \alpha)}=\frac{\alpha}{1+\alpha(1-1 / \alpha)}=\frac{\alpha}{\alpha}=1 .
$$

As in the proof of Lemma 4.7, there exists a unique $l_{\max }(\tau)=\sqrt{\alpha-1 / s_{+}^{2}(\tau)}>0$ such that $h_{2}\left(l_{\max }(\tau), \tau\right)=1$. Then $l_{\max }(\tau)<z_{2}(\tau)$. Therefore, $h_{1}(\omega, \tau)>0$ for any $\omega \in\left[0, l_{\max }(\tau)\right]$. The rest of the proof is similar to the proof of Lemma 4.7. Furthermore, $\theta_{1}(\tau)<\pi$, since $h_{2}\left(\omega_{1}(\tau), \tau\right)>-1$ for any $\omega_{1}(\tau) \in\left[0, l_{\max }(\tau)\right)$. 
Theorem 4.9. Consider system (2.3) with $D=\Delta=1$.

(1) Suppose $\alpha>(7+3 \sqrt{5}) / 2, k>\alpha /(\sqrt[4]{\alpha}-1)$, and $\tau \in I_{1}$ given by (4.22). For $\tau \in I_{1}$ and $j=1,2, \omega_{j}(\tau)$ is nonnegative and there exists $\epsilon_{j}>0$ and $\theta_{j}(\tau)$ such that $\epsilon_{j} \leqslant \theta_{j}(\tau) \leqslant \pi$ and $\theta_{j}(\tau)$ satisfies (4.56). If there exists $n \geqslant 0$ such that $\theta_{j}(\tau)+2 n \pi$ intersects $\tau \omega_{j}(\tau)$ at some $\tau_{n}^{j} \in I_{1}$, then (4.4) has a pair of pure imaginary eigenvalues $\lambda= \pm i \omega_{j}\left(\tau_{n}^{j}\right)$. System (2.3) undergoes a Hopf bifurcation at $\tau_{n}^{j}$ provided $\mathrm{d} \operatorname{Re}(\lambda(\tau)) /\left.\mathrm{d} \tau\right|_{\tau=\tau_{n}^{j}} \neq 0$.

(2) Suppose $\alpha>1, k>\alpha(1 /(1 / 4+\sqrt{1 / 16+1 /(2 \alpha)})-1)^{-1}$, and $\tau \in I_{2}$ given by (4.41). For $\tau \in I_{2}$, only $\omega_{1}(\tau)$ is positive and there exists $\epsilon>0$ and $\theta_{1}(\tau)$ such that $\epsilon \leqslant \theta_{1}(\tau)<\pi$ and $\theta_{1}(\tau)$ satisfies (4.56) for $j=1$. If there exists $n \geqslant 0$ such that $\theta_{1}(\tau)+2 n \pi$ intersects $\tau \omega_{1}(\tau)$ at some $\tau_{n}^{1} \in I_{2}$, then (4.4) has a pair of pure imaginary eigenvalues $\lambda= \pm i \omega_{1}\left(\tau_{n}^{1}\right)$. System (2.3) undergoes a Hopf bifurcation at $\tau_{n}^{1}$ provided $\mathrm{d} \operatorname{Re}(\lambda(\tau)) /\left.\mathrm{d} \tau\right|_{\tau=\tau_{n}^{1}} \neq 0$.

Proof. Assume $D=\Delta=1$ in system (2.3).

Case 1. Suppose $\tau \in I_{1}$. By Theorem 4.5, $\omega_{j}(\tau) \geqslant 0$ for $j=1$, 2. By Lemma 4.7, there exists $\epsilon_{j}>0$ and $\theta_{j}(\tau)$ such that $\epsilon_{j} \leqslant \theta_{j}(\tau) \leqslant \pi$ and $\theta_{j}(\tau)$ satisfies (4.56). Assume that there exists a positive integer $\tau_{n}^{j} \in I_{1}$ such that $\theta_{j}\left(\tau_{n}^{j}\right)+2 n \pi=\tau_{n}^{j} \omega_{j}\left(\tau_{n}^{j}\right)$ for some integer $n \geqslant 0$. Then system (4.52) has one solution $\left(\tau_{n}^{j}, \omega_{j}\left(\tau_{n}^{j}\right)\right)$. Equation (4.4) has a pair of pure imaginary eigenvalues $\lambda= \pm i \omega_{j}\left(\tau_{n}^{j}\right)$.

In what follows, we show that the conditions required for a Hopf Bifurcation (see Theorem A.1 in the appendix) are satisfied by the linearization (3.3) of (2.3) at $E_{+}$. In (A.1), choosing $\tau$ as the bifurcation parameter and letting

$$
\begin{aligned}
D\left(\tau, z_{t}\right) & =\left[\begin{array}{lll}
1 & 0 & 0 \\
0 & 1 & 0 \\
0 & 0 & 1
\end{array}\right]\left[\begin{array}{l}
z_{1}(t) \\
z_{2}(t) \\
z_{3}(t)
\end{array}\right], \\
L\left(\tau, z_{t}\right) & =\left[\begin{array}{ccc}
-1-\alpha x_{+} & -\alpha s_{+} & 0 \\
\alpha x_{+} & -D+\alpha s_{+}-k y_{+} & -k x_{+} \\
0 & 0 & -\Delta
\end{array}\right]\left[\begin{array}{l}
z_{1}(t) \\
z_{2}(t) \\
z_{3}(t)
\end{array}\right]+\left[\begin{array}{ccc}
0 & 0 & 0 \\
0 & 0 & 0 \\
0 & k e^{\Delta \tau} y_{+} & k e^{\Delta \tau} x_{+}
\end{array}\right]\left[\begin{array}{l}
z_{1}(t-\tau) \\
z_{2}(t-\tau) \\
z_{3}(t-\tau)
\end{array}\right],
\end{aligned}
$$

the linearization (3.3) of (2.3) at $E_{+}$is of the form (A.1). Taking $a$ to be any positive real number and $b=1 / 2$, hypothesis $\left(S_{1}\right)$ in the Hopf Bifurcation Theorem holds, since

$$
\begin{gathered}
\left|\operatorname{det}\left(\sum_{k=0}^{\infty} A_{k}(\alpha) e^{-\lambda r_{k}(\alpha)}\right)\right|=\left|\operatorname{det}\left[\begin{array}{lll}
1 & 0 & 0 \\
0 & 1 & 0 \\
0 & 0 & 1
\end{array}\right]\right|=1 \geqslant \frac{1}{2}, \\
\left|\operatorname{det}\left(\sum_{k=0}^{\infty} A_{k}(\alpha) e^{-\lambda r_{k}(\alpha)}+\int_{-1}^{0} A(\alpha, \theta) e^{\lambda \theta} \mathrm{d} \theta\right)\right|=\left|\operatorname{det}\left[\begin{array}{lll}
1 & 0 & 0 \\
0 & 1 & 0 \\
0 & 0 & 1
\end{array}\right]\right|=1 \geqslant \frac{1}{2}
\end{gathered}
$$

for all $\tau \in \mathbb{R}$ and $|\operatorname{Re} \lambda|<a$. 
The characteristic equation (4.4) of (3.3) at $E_{+}$has a pair of pure imaginary eigenvalues $\lambda= \pm \omega_{1}\left(\tau_{n}^{j}\right)$ and no other root of (4.4) is an integral multiple of $\pm \omega_{1}\left(\tau_{n}^{j}\right)$. Hence the hypothesis $\left(S_{2}\right)$ in the Hopf Bifurcation Theorem holds. Therefore, (2.3) undergoes a Hopf bifurcation at $E_{+}$when $\tau=\tau_{n}^{j}$ provided $\mathrm{d} \operatorname{Re}(\lambda(\tau)) /\left.\mathrm{d} \tau\right|_{\tau=\tau_{n}^{j}} \neq 0$.

Case 2. Suppose $\tau \in I_{2}$. By Theorem 4.5, only $\omega_{1}(\tau)>0$. By Lemma 4.8, there exists $\epsilon>0$ and $\theta_{1}(\tau)$ such that $\epsilon \leqslant \theta_{1}(\tau)<\pi$ and $\theta_{1}(\tau)$ satisfies (4.56). Assume there exists $\tau_{n}^{1} \in I_{2}$ such that $\theta_{1}\left(\tau_{n}^{1}\right)+2 n \pi=\tau_{n}^{1} \omega_{1}\left(\tau_{n}^{1}\right)$ for some integer $n \geqslant 0$. Then system (4.52) has one solution $\left(\tau_{n}^{1}, \omega_{1}\left(\tau_{n}^{1}\right)\right)$. Equation (4.4) has a pair of pure imaginary eigenvalues $\lambda= \pm i \omega_{1}\left(\tau_{n}^{1}\right)$. The rest of the proof is similar to that of Case 1 when $j=1$.

Corollary 4.10. Consider system (2.3) with $D=\Delta=1$.

(1) Suppose $\alpha>(7+3 \sqrt{5}) / 2, k>\alpha /(\sqrt[4]{\alpha}-1)$, and $\tau \in I_{1}$ given by (4.22). For $\tau \in I_{1}$, $\omega_{j}(\tau)$ is nonnegative and there exists $\epsilon_{j}>0$ and $\theta_{j}(\tau)$ such that $\epsilon_{j} \leqslant \theta_{j}(\tau) \leqslant \pi$ and $\theta_{j}(\tau)$ satisfies (4.56) for $j=1$, 2. If there exists a positive integer $n_{j} \geqslant 0$ such that $\min _{\tau \in I_{1}} \tau \omega_{j}(\tau) \leqslant 2 n_{j} \pi$ and $\max _{\tau \in I_{1}} \tau \omega_{j}(\tau)>\left(2 n_{j}+1\right) \pi$, then $\theta_{j}(\tau)+2 n_{j} \pi$ intersects $\tau \omega_{j}(\tau)$ at least once at some $\tau_{n_{j}}^{j} \in I_{1}$. System (2.3) undergoes a Hopf bifurcation at $\tau_{n_{j}}^{j}$ provided $\mathrm{d} \operatorname{Re}(\lambda(\tau)) /\left.\mathrm{d} \tau\right|_{\tau=\tau_{n_{j}}^{j}} \neq 0$.

(2) Suppose $\alpha>1, k>\alpha(1 /(1 / 4+\sqrt{1 / 16+1 /(2 \alpha)})-1)^{-1}$, and $\tau \in I_{2}$ defined in (4.41). For $\tau \in I_{2}$, only $\omega_{1}(\tau)$ is positive. There exists $\epsilon>0$ and $\theta_{1}(\tau)$ such that $\epsilon \leqslant \theta_{1}(\tau)<\pi$ and $\theta_{1}(\tau)$ satisfies (4.56) for $j=1$. If there exists a positive integer $N \geqslant 0$ such that $\max _{\tau \in I_{2}} \tau \omega_{1}(\tau)>(2 N+1) \pi$, then for any $0 \leqslant n \leqslant N, \theta_{1}(\tau)+2 n \pi$ intersects $\tau \omega_{1}(\tau)$ at least once at some $\tau_{n}^{1} \in I_{2}$. System (2.3) undergoes a Hopf bifurcation at $\tau_{n}^{1}$ provided $\mathrm{d} \operatorname{Re}(\lambda(\tau)) /\left.\mathrm{d} \tau\right|_{\tau=\tau_{n}^{1}} \neq 0$.

Proof. Assume $D=\Delta=1$ in system (2.3).

Case 1. Suppose $\tau \in I_{1}$. By Theorem 4.5, $\omega_{j}(\tau) \geqslant 0$ for $j=1,2$. By Lemma 4.7, there exists $\epsilon_{j}>0$ and $\theta_{j}(\tau)$ such that $\epsilon_{j} \leqslant \theta_{j}(\tau) \leqslant \pi$ and $\theta_{j}(\tau)$ satisfies (4.56). Assume that there exists a positive integer $n_{j} \geqslant 0$ such that $\min _{\tau \in I_{1}} \tau \omega_{j}(\tau) \leqslant 2 n_{j} \pi$ and $\max _{\tau \in I_{1}} \tau \omega_{j}(\tau)>\left(2 n_{j}+1\right) \pi$. For such $n_{j}$,

$$
\min _{\tau \in I_{1}} \tau \omega_{j}(\tau)<\epsilon_{j}+2 n_{j} \pi \leqslant \theta_{j}(\tau)+2 n_{j} \pi \leqslant\left(2 n_{j}+1\right) \pi<\max _{\tau \in I_{1}} \tau \omega_{j}(\tau)
$$

By the Mean Value Theorem, there exists $\tau_{n_{j}}^{j} \in I_{1}$ such that $\theta_{j}\left(\tau_{n_{j}}^{j}\right)+2 n_{j} \pi=\tau_{n_{j}}^{j} \omega_{j}\left(\tau_{n_{j}}^{j}\right)$. By Theorem 4.9. Case 1, the conclusion follows.

Case 2. Suppose $\tau \in I_{2}$. By Theorem 4.5, only $\omega_{1}(\tau)>0$. By Lemma 4.8, there exists $\epsilon>0$ and $\theta_{1}(\tau)$ such that $\epsilon \leqslant \theta_{1}(\tau)<\pi$ and $\theta_{1}(\tau)$ satisfies (4.56). Assume that there exists a positive integer $N \geqslant 0$ such that $\max _{\tau \in I_{2}} \tau \omega_{1}(\tau)>(2 N+1) \pi$. By $(4.41), 0 \in I_{2}$. Therefore $\min _{\tau \in I_{2}} \tau \omega_{1}(\tau)=0$. For $0 \leqslant n \leqslant N$,

$$
\min _{\tau \in I_{2}} \tau \omega_{1}(\tau)<\epsilon+2 n \pi \leqslant \theta_{i}(\tau)+2 n \pi \leqslant(2 n+1) \pi<\max _{\tau \in I_{2}} \tau \omega_{1}(\tau)
$$


By the Mean Value Theorem, there exists $\tau_{n}^{1} \in I_{2}$ such that $\theta_{1}\left(\tau_{n}^{1}\right)+2 n \pi=\tau_{n}^{1} \omega_{1}\left(\tau_{n}^{1}\right)$. By Theorem 4.9. Case 2, the conclusion follows.

Corollary 4.11. Consider system (2.3) with $D=\Delta=1$. Assume $\alpha>(7+3 \sqrt{5}) / 2$ and $k>\alpha /(\sqrt[4]{\alpha}-$ 1). If $1 / 4+\sqrt{1 / 16+1 /(2 \alpha)}>k /(\alpha+k)$, then $I_{1}=[0, \ln ((k / \alpha)(\sqrt[4]{\alpha}-1))]$, where $I_{1}$ was defined in (4.22). For any $\tau \in I_{1}, \omega_{j}(\tau)$ is nonnegative and there exists $\epsilon_{j}>0$ and $\theta_{j}(\tau)$ such that $\epsilon_{j} \leqslant \theta_{j}(\tau) \leqslant$ $\pi$ and $\theta_{j}(\tau)$ satisfies (4.56) for $j=1,2$. If there exists a positive integer $N_{j} \geqslant 0(j=1,2)$ such that $\max _{\tau \in I_{1}} \tau \omega_{j}(\tau)>\left(2 N_{j}+1\right) \pi$, then for any $0 \leqslant n \leqslant N_{j}, \theta_{j}(\tau)+2 n \pi$ intersects $\tau \omega_{j}(\tau)$ at least once at some $\tau_{n}^{j} \in I_{1}$. System (2.3) undergoes a Hopf bifurcation at $\tau_{n}^{j}$ provided $\mathrm{d} \operatorname{Re}(\lambda(\tau)) /\left.\mathrm{d} \tau\right|_{\tau=\tau_{n}^{j}} \neq 0$.

Proof. Assume $1 / 4+\sqrt{1 / 16+1 /(2 \alpha)}>k /(\alpha+k)$. Then $\ln ((k / \alpha)(1 /(1 / 4+\sqrt{1 / 16+1 /(2 \alpha)})-$ $1))<0$. By $(4.22)$,

$$
\begin{aligned}
I_{1} & =\left[\max \left\{0, \ln \left(\frac{k}{\alpha}\left(\frac{1}{1 / 4+\sqrt{1 / 16+1 /(2 \alpha)}}-1\right)\right)\right\}, \ln \left(\frac{k(\sqrt[4]{\alpha}-1)}{\alpha}\right)\right] \\
& =\left[0, \ln \left(\frac{k}{\alpha}(\sqrt[4]{\alpha}-1)\right)\right] .
\end{aligned}
$$

For any $\tau \in I_{1}$, by Theorem 4.5, $\omega_{j}(\tau) \geqslant 0$ for $j=1,2$. By Lemma 4.7, there exists $\epsilon_{j}>0$ and $\theta_{j}(\tau)$ such that $\epsilon_{j} \leqslant \theta_{j}(\tau) \leqslant \pi$ and $\theta_{j}(\tau)$ satisfies (4.56). Noting $0 \in I_{1}, \min _{\tau \in I_{1}} \tau \omega_{j}(\tau)=0$. Assume there exists a positive integer $N_{j} \geqslant 0(j=1,2)$ such that $\max _{\tau \in I_{1}} \tau \omega_{j}(\tau)>\left(2 N_{j}+1\right) \pi$. For any $0 \leqslant n \leqslant N_{j}, \min _{\tau \in I_{1}} \tau \omega_{j}(\tau)=0 \leqslant 2 n \pi$ and $\max _{\tau \in I_{1}} \tau \omega_{j}(\tau)>\left(2 N_{j}+1\right) \pi \geqslant(2 n+1) \pi$. By Corollary 4.10, the conclusion follows.

\section{Numerical Results}

This section includes bifurcation diagrams involving the interior equilibrium $E_{+}$and numerical simulations of periodic solutions of the predator-prey model in the chemostat.

\subsection{Variation of Eigenvalues}

To study the stability switches of $E_{+}$, DDEBIFTOOL (see [11, 12]) was chosen to illustrate how the real part of the eigenvalues of (4.2) changes as parameters $\alpha$ and $\tau$ vary.

First fix parameters $D=\Delta=1, k=24$, and $\tau=0.5$. Taking $\alpha$ as the bifurcation parameter and varying it from 0 to 10 , the real part of the eigenvalues with largest real part of (4.2) was plotted in Figure 1. At $\alpha \approx 1.15$ and $\alpha \approx 1.5$, there is either a zero eigenvalue or a pair of pure imaginary roots. For $\alpha \in(1.15,1.5)$, all eigenvalues have negative real parts. For example, taking $\alpha=1.3$, Figure 2(a) shows that the eigenvalues of (4.2) with largest real parts (the ones in the circle) have negative real parts. Note that due to the scaling, the eigenvalues in the circle seem to be indistinguishable from zero. But in fact, they are a pair of complex eigenvalues with real parts slightly less than zero. DDEBIFTOOL can keep track of the occurrence of a pair of pure imaginary eigenvalues as $\alpha$ varies in the neighborhood of $\alpha=$ 1.5. Figure 2(b) clearly shows that there is a pair of pure imaginary eigenvalues. Hence, Hopf bifurcation is possible. Note that by continuation, the pair of eigenvalues with largest real parts in Figure 2(a) for $\alpha=1.3$ becomes the pair of pure imaginary eigenvalues in Figure 2(b) for $\alpha \approx 1.5$. 


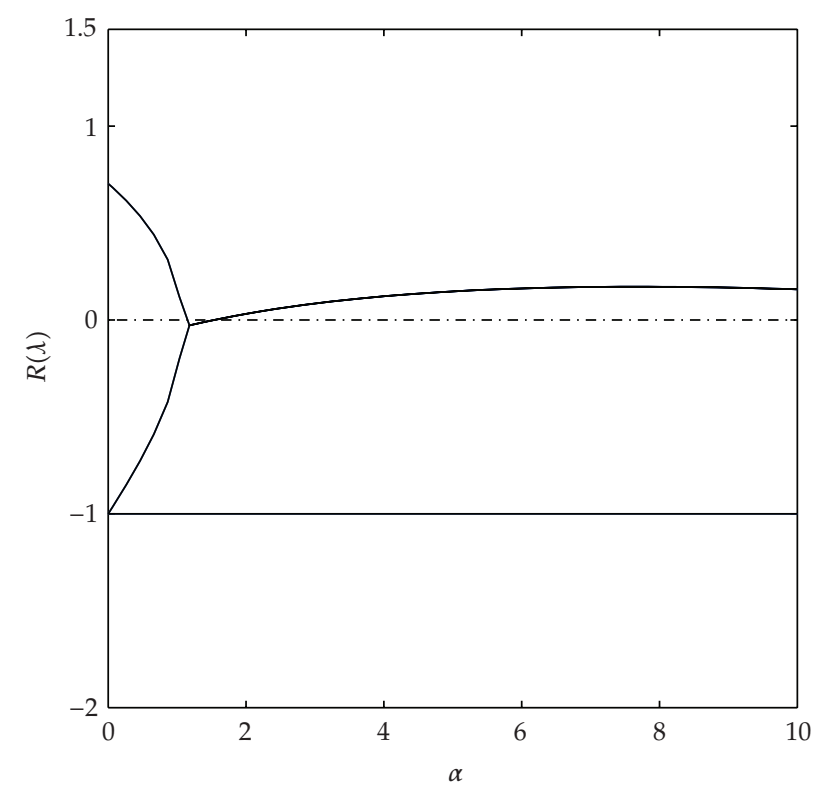

Figure 1: Variation of the largest real part of the eigenvalues as the bifurcation parameter $\alpha$ is varied. At $\alpha \approx 1.15$ and 1.5 , the largest real part crosses zero and it seems that there is a zero eigenvalue for $\alpha \approx 1.15$ and a pair of pure imaginary eigenvalues for $\alpha \approx 1$. The largest real part becomes positive as $\alpha$ increases through 1.5. But as $\alpha$ increases further, for $\alpha \approx 17$, the largest real part crosses zero again and remains negative thereafter. There is a second Hopf bifurcation at $\alpha \approx 17$. This is consistent with what is observed in Figure 3 when $\tau=0.5$ and $\alpha$ varies from 0 to 30 . Parameters are $D=\Delta=1, k=24$, and $\tau=0.5$.

Finally fix all parameters as before and vary both $\tau$ and $\alpha$. In Figure 3, we plot the Hopf bifurcation diagram in $\alpha$ and $\tau$ parameter space. The curve at the left upper corner is $\tau=\tau_{c}$. For any pair $(\alpha, \tau)$ below that curve, a coexistence equilibrium $E_{+}$exists (i.e., all components are positive). For any pair $(\alpha, \tau)$ on the closed curve, there is a Hopf bifurcation. Inside the closed curve, there is a periodic solution surrounding $E_{+}$. For any $(\alpha, \tau)$ outside the closed curve and below $\tau=\tau_{c}$, the coexistence equilibrium $E_{+}$is stable.

\subsection{Simulations Demonstrating Hopf Bifurcations}

In this section, we illustrate Theorem 4.9 for system (2.3). Take $D=\Delta=1$ and let $\tau$ vary. We choose parameters $\alpha=100$ and $k=100$ for Case 1 (see Figures 4-12), and $\alpha=2$ and $k=20$ for Case 2 (see Figures 13-18).

Case 1. Note that $I_{1}$ is given by (4.22). Since $\ln ((k / \alpha)(1 /(1 / 4+\sqrt{1 / 16+1 /(2 \alpha)})-1))=-0.03$,

$$
\max \left\{0, \ln \left(\frac{k}{\alpha}\left(\frac{1}{1 / 4+\sqrt{1 / 16+1 /(2 \alpha)}}-1\right)\right)\right\}=0
$$

Also, $\ln (k(\sqrt[4]{\alpha}-1) / \alpha) \approx 0.77$. Therefore $I_{1} \approx[0,0.77]$. By Theorem $4.9, \omega_{j}(\tau)$ is positive and $\theta_{j}(\tau)$ satisfies (4.56) for any $\tau \in I_{1}$ and $j=1,2$. 

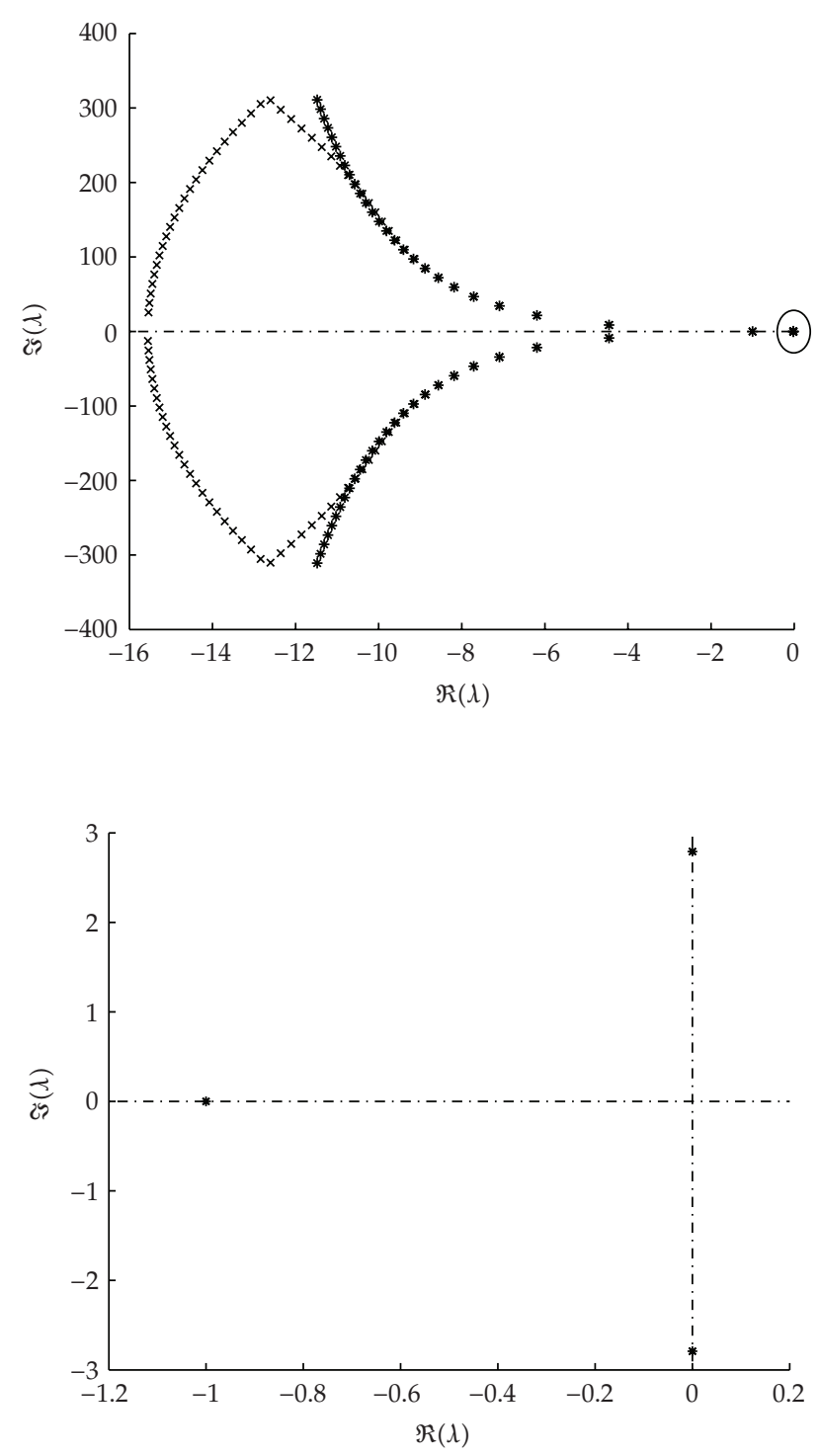

Figure 2: Eigenvalues with the largest real parts of the characteristic equation (4.2) at $E_{+}$. Parameters are the same as in Figure 1 except $\alpha=1.3$ for the TOP and $\alpha \approx 1.5$ for the BOTTOM graph. Due to the scaling, the eigenvalues in the circle in the TOP graph seem indistinguishable from zero. In fact, they are a pair of complex eigenvalues with real parts slightly less than zero. As $\alpha$ varies from 1.3 to 1.5 , the pair of complex eigenvalues with largest real part becomes a pair of pure imaginary roots in the BOTTOM graph. The eigenvalue with the second largest real part remains equal to -1 . This is consistent with our analytical results that showed that (4.2) has a constant eigenvalue -1 when $D=\Delta=$ 1.

Figure 4 shows that $\theta_{j}(\tau)$ intersects $\tau \omega_{j}(\tau)$ at some $\tau_{0}^{j}$ with $\tau_{0}^{1} \approx 0.022$ and $\tau_{0}^{2} \approx 0.48$. We see that $\theta_{j}(\tau)+2 n \pi$ has no intersection with $\tau \omega_{j}(\tau)$ for $n \geqslant 2$ and $j=1$,2. By Theorem 4.9, (4.4) has two distinct pairs of pure imaginary eigenvalues $\lambda= \pm i \omega_{j}\left(\tau_{0}^{j}\right)$. Next we need to check if $\left.\operatorname{Re}(\mathrm{d} \lambda(\tau) / \mathrm{d} \tau)\right|_{\tau=\tau_{0}^{j}} \neq 0$. 


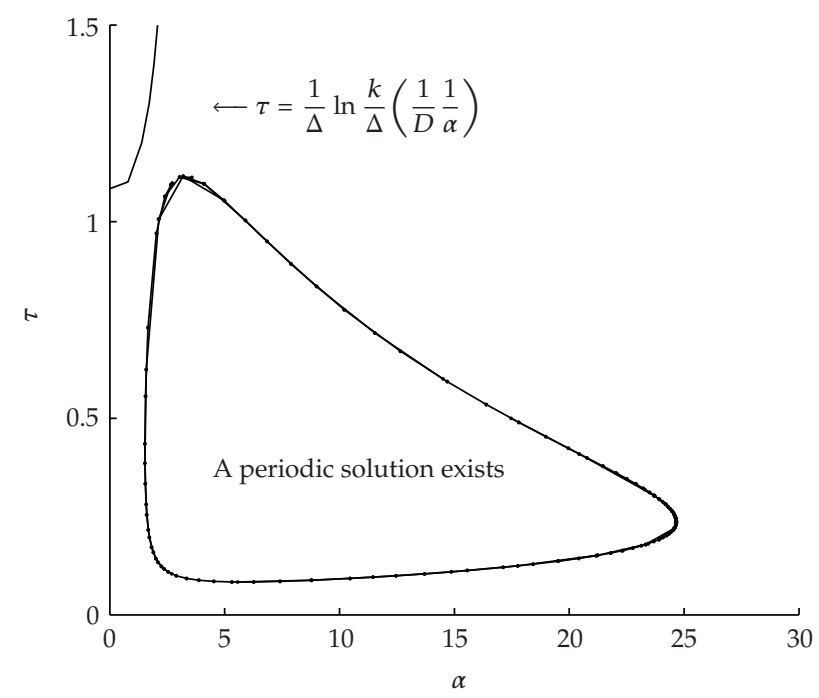

Figure 3: The two-parameter bifurcation diagram of $E_{+}$in $\tau$ and $\alpha$ parameter space. Parameters are the same as in Figure 1 (i.e., $D=\Delta=1, k=24$ ) except now both $\tau$ and $\alpha$ are allowed to vary. For any pair $(\alpha, \tau)$ on the closed curve, there is a Hopf bifurcation of $E_{+}$. Inside the closed curve, there is a periodic solution surrounding $E_{+}$. For any $(\alpha, \tau)$ outside the closed curve and below $\tau=\tau_{c}$, the coexistence equilibrium $E_{+}$ is stable.

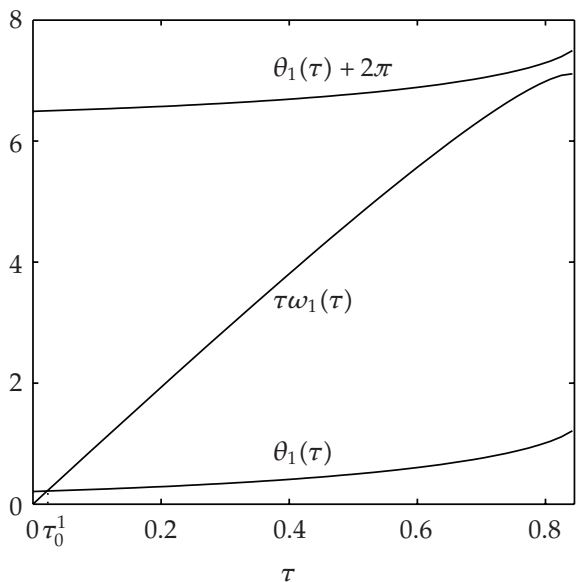

(a)

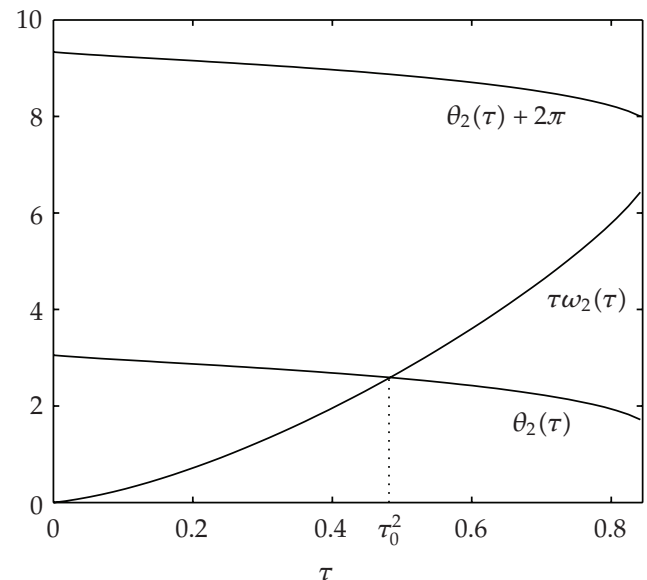

(b)

Figure 4: Critical value of delay $\tau$ at which a Hopf Bifurcation occurs for $D=\Delta=1, k=100, \alpha=100$.

As in Beretta and Kuang [13], we can define

$$
S_{n}^{j}(\tau)=\tau-\frac{\theta_{j}(\tau)+2 n \pi}{\omega_{j}(\tau)}, \quad \text { for } j=1,2, n=0,1,2 \ldots
$$

Any zero $\tau_{n}^{j}$ of $S_{n}^{j}(\tau)$ is an intersection of $\theta_{j}(\tau)+2 n \pi$ and $\tau \omega_{j}(\tau)$ and vice versa. By (4.10) in Beretta and Kuang [13] and noting that $\left(q^{2}-p^{2}(\tau)+2 \alpha^{2}(\tau)\right)^{2}-4\left(\alpha^{2}(\tau)-c^{2}(\tau)\right)>0$, we have 


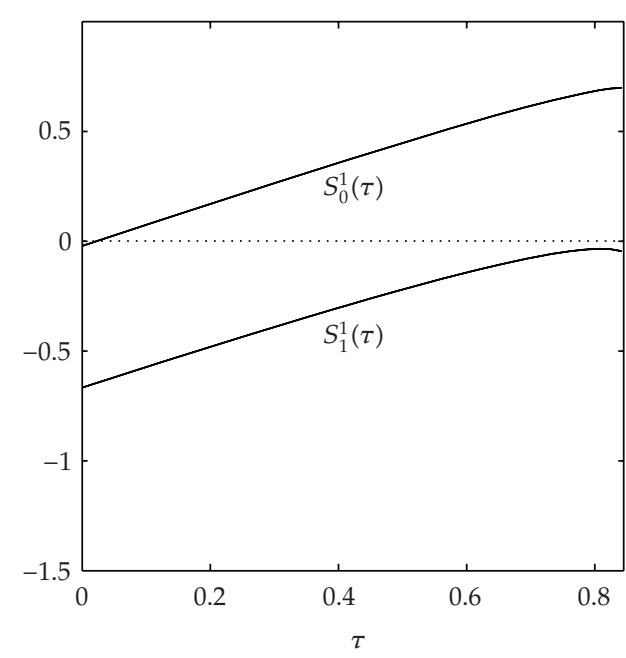

(a)

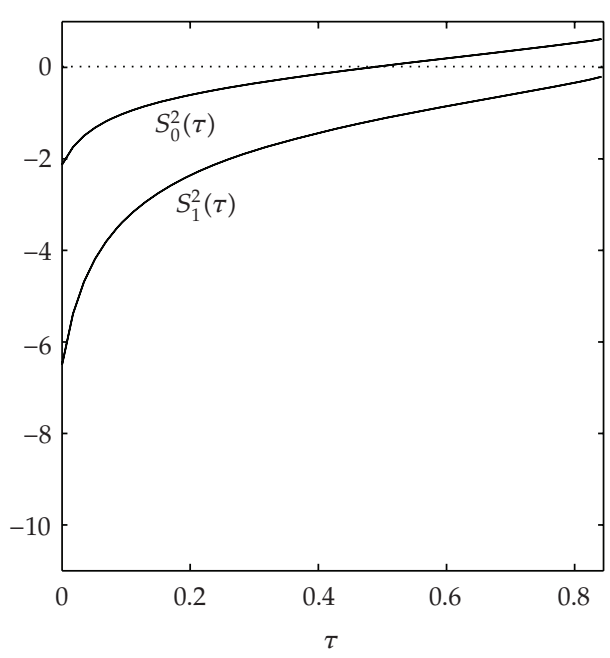

(b)

Figure 5: If the slope of $S_{n}^{j}(\tau)$ is nonzero at $\tau$ when $S_{n}^{j}(\tau)=0(j=1,2)$, the transversality condition holds and there is a Hopf bifurcation. The other parameters are the same as for Figure 4.

the relation

$$
\begin{aligned}
& \operatorname{sign}\left\{\left.\operatorname{Re} \frac{\mathrm{d} \lambda(\tau)}{\mathrm{d} \tau}\right|_{\tau=\tau_{n}^{j}}\right\} \\
& = \pm \operatorname{sign}\left\{\left(q^{2}-p^{2}(\tau)+2 \alpha^{2}(\tau)\right)^{2}-4\left(\alpha^{2}(\tau)-c^{2}(\tau)\right)\right\} \operatorname{sign}\left\{\left.\frac{\mathrm{d} S_{n}^{j}(\tau)}{\mathrm{d} \tau}\right|_{\tau=\tau_{n}^{j}}\right\} \\
& = \pm \operatorname{sign}\left\{\left.\frac{\mathrm{d} S_{n}^{j}(\tau)}{\mathrm{d} \tau}\right|_{\tau=\tau_{n}^{j}}\right\},
\end{aligned}
$$

where we take + for $j=1$ and - for $j=2$.

From Figure 5(a), it is observed that $S_{n}^{1}$ has only one zero $\tau_{0}^{1} \approx 0.022$ at $n=0$ with $\operatorname{sign}\left\{\mathrm{d} S_{0}^{1}(\tau) /\left.\mathrm{d} \tau\right|_{\tau=\tau_{0}^{1}}\right\}>0$. Hence, $\operatorname{sign}\left\{\left.\operatorname{Re}(\mathrm{d} \lambda(\tau) / \mathrm{d} \tau)\right|_{\tau=\tau_{0}^{1}}\right\}>0$. By Theorem 4.9, system $(2.3)$ undergoes a Hopf bifurcation at $\tau_{0}^{1}$. Similarly from Figure $5(\mathrm{~b}), S_{n}^{2}$ has only one zero $\tau_{0}^{2} \approx 0.48$ for $n=0$ and (2.3) undergoes a Hopf bifurcation at $\tau_{0}^{2}$.

Next we used MATLAB to simulate solutions of model (2.3) for several values of $\tau$. For each fixed delay $\tau$, we chose initial data $s(t)=s_{+}(\tau)-0.01, x(t)=x_{+}(\tau)+0.01$, and $y(t)=y_{+}(\tau)+0.001$ for $t \in[-\tau, 0]$. From Figure 6 , we can see that the equilibrium $E_{+}$is stable if $\tau=0.02<\tau_{0}^{1}$. As delay $\tau$ increases past $\tau_{0}^{1} \approx 0.022$, where a Hopf bifurcation occurs, a pair of complex eigenvalues of (4.4) enters the right-half plane. The equilibrium $E_{+}$loses its stability and a periodic solution bifurcates from $E_{+}$(see Figures 7 and 8 ). As we increase the delay further to $\tau=0.4<\tau_{0}^{2}$, the periodic solution still exists and remains stable (see Figures 9 and 10). However, as the delay $\tau$ increases further, past $\tau_{0}^{2}$, the stable periodic solution 


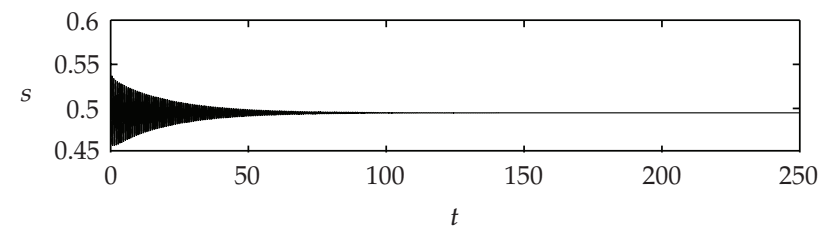

(a)

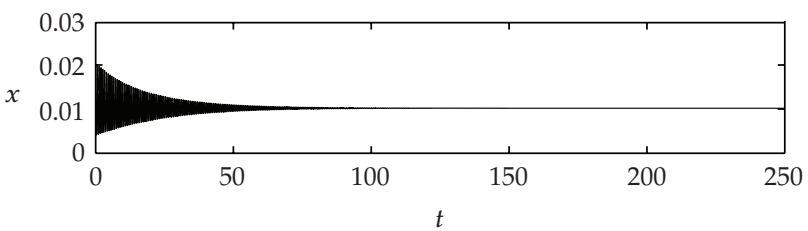

(b)

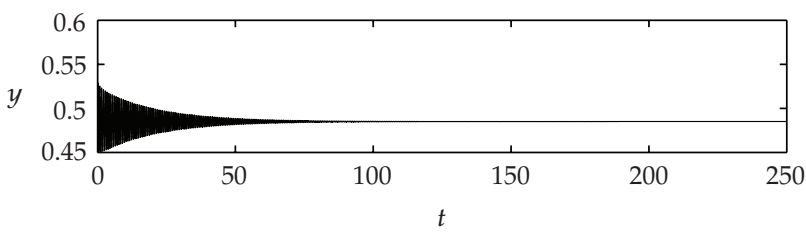

(c)

Figure 6: Equilibrium $E_{+}(\tau)$ is stable when $\tau=0.02<\tau_{0}^{1}$. The other parameters are the same as for Figure 4 .

$s$

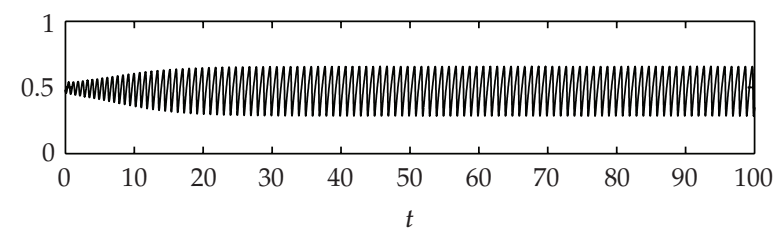

(a)

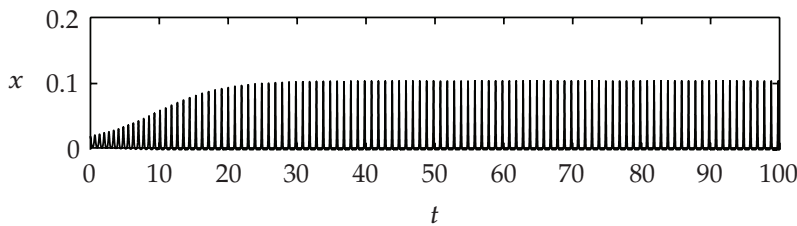

(b)

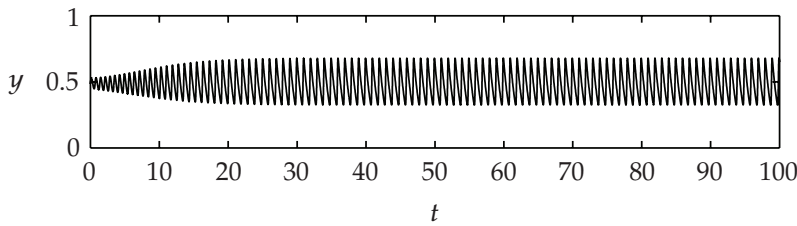

(c)

Figure 7: Time series of a periodic solution, for $\tau=0.03$. The other parameters are the same as for Figure 4 . 


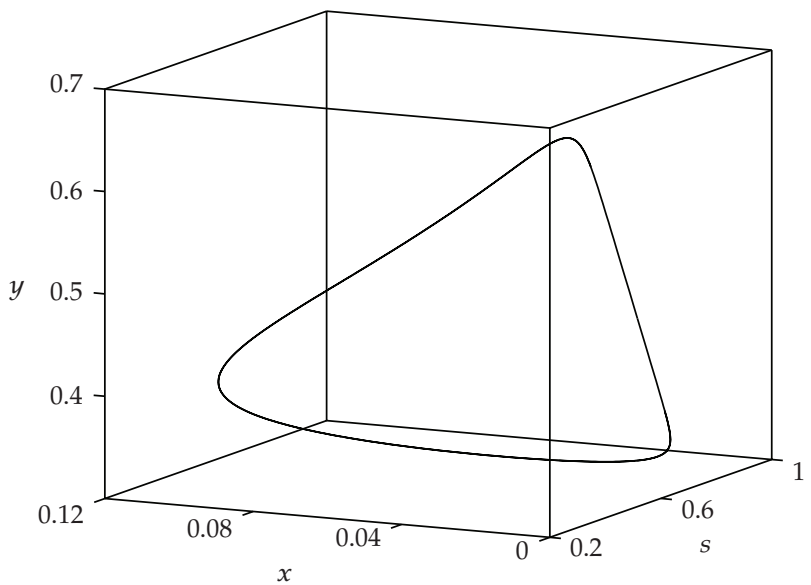

Figure 8: The trajectory in phase space of the periodic solution in Figure 7 for $\tau=0.03$.

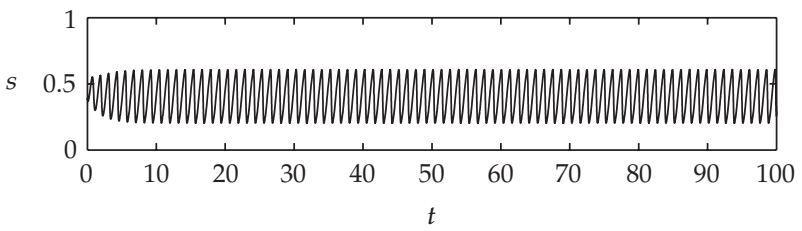

(a)

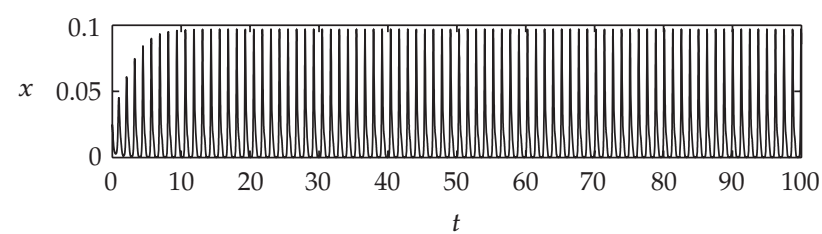

(b)

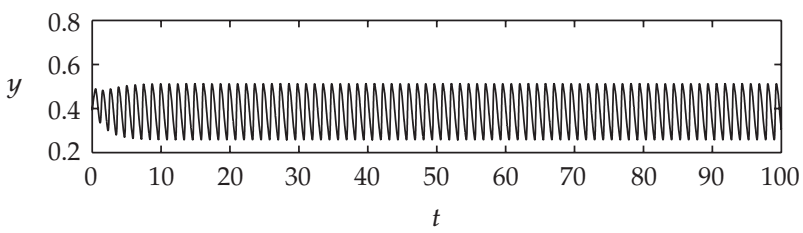

(c)

Figure 9: Time series of a periodic solution for $\tau=0.4$. The other parameters are the same as for Figure 4 .

disappears in a second Hopf bifurcation, and $E_{+}$regains stability (see Figure 11). We provide a bifurcation diagram illustrating the change in dynamics as $\tau$ varies (see Figure 12). For any $\tau \in\left(\tau_{0}^{1}, \tau_{0}^{2}\right)$, there is an orbitally asymptotically stable periodic solution.

Case 2. Take $k=20$ and $\alpha=2$. For such parameters, $I_{2} \approx[0,0.85]$. By Theorem $4.9, \omega_{1}(\tau)$ is positive and $\theta_{1}(\tau)$ satisfies (4.56) for $j=1$ and $\tau \in I_{2}$. Figure 13 shows that $\theta_{1}(\tau)$ intersects $\tau \omega_{1}(\tau)$ twice. To distinguish these intersections, denote them as $\tau_{0,1}^{1} \approx 0.2$ and $\tau_{0,2}^{1} \approx 0.7$. On the other hand, $\theta_{1}(\tau)+2 \pi$ has no intersection with $\tau \omega_{1}(\tau)$. 


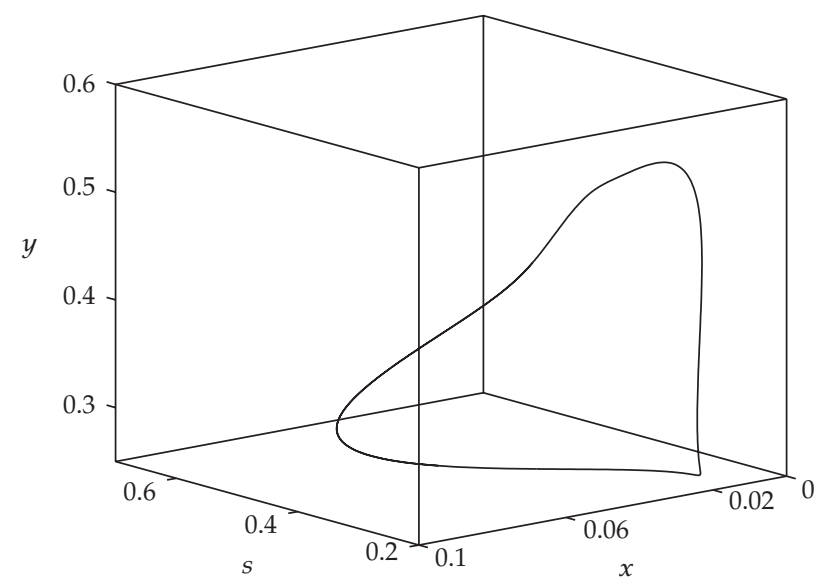

Figure 10: The trajectory in phase space of the periodic solution shown in Figure 9.

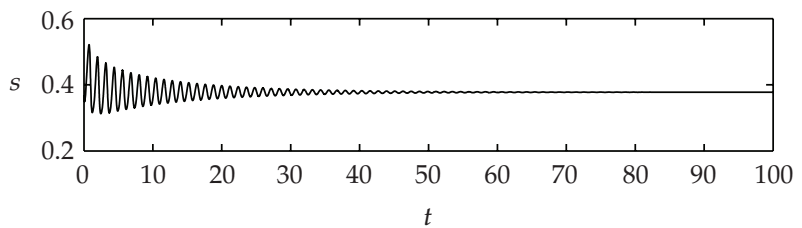

(a)

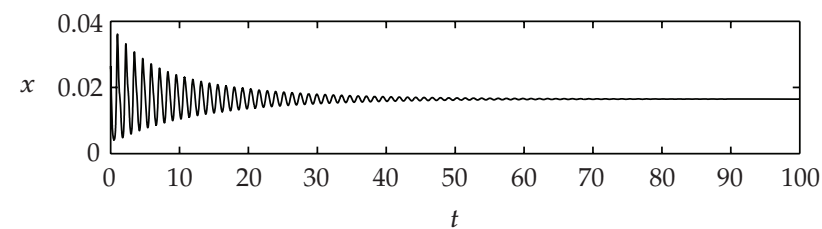

(b)

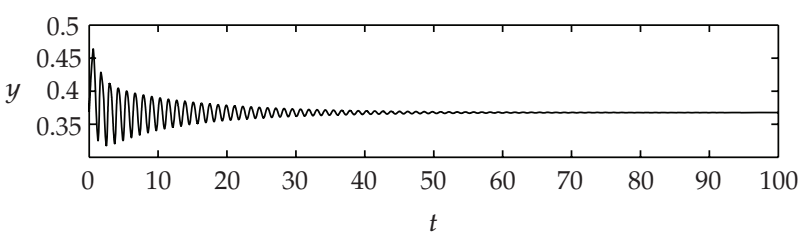

(c)

Figure 11: The periodic solution disappears at the secondary Hopf bifurcation at $\tau_{0}^{2} \approx 0.48$ and $E_{+}$regains stability. In this figure $\tau=0.5>\tau_{0}^{2}$. The other parameters are the same as for Figure 4 .

From Figure 14,

$$
\operatorname{sign}\left\{\left.\frac{\mathrm{d} S_{0}^{1}(\tau)}{\mathrm{d} \tau}\right|_{\tau=\tau_{0,1}^{1}}\right\}>0, \quad \operatorname{sign}\left\{\left.\frac{\mathrm{d} S_{1}^{1}(\tau)}{\mathrm{d} \tau}\right|_{\tau=\tau_{0,2}^{1}}\right\}<0
$$




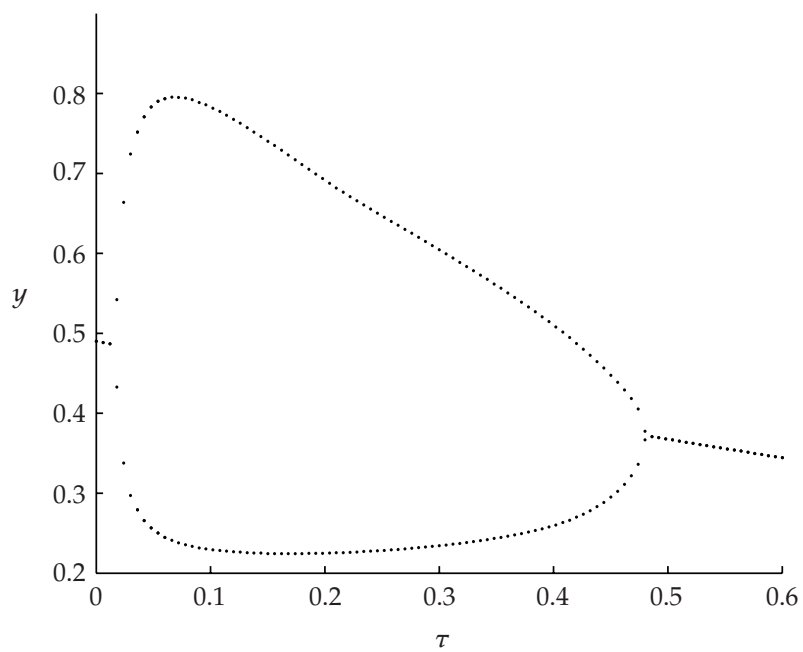

Figure 12: Bifurcation diagram as the delay varies. The maximum and minimum amplitude of the $y$ component of the solution is plotted on the ordinate axis. Parameters are the same as for Figure 4.

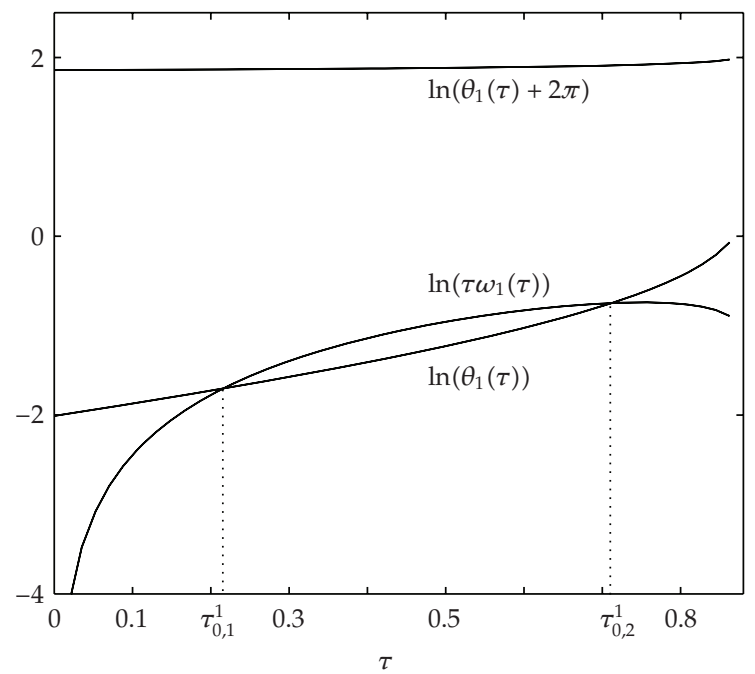

Figure 13: Intersections indicate critical values of the delay at which Hopf bifurcations occur. Parameters are $D=\Delta=1, k=20, \alpha=2$.

By (5.3),

$$
\operatorname{sign}\left\{\left.\operatorname{Re} \frac{\mathrm{d} \lambda(\tau)}{\mathrm{d} \tau}\right|_{\tau=\tau_{0,1}^{1}}\right\}>0, \quad \operatorname{sign}\left\{\left.\operatorname{Re} \frac{\mathrm{d} \lambda(\tau)}{\mathrm{d} \tau}\right|_{\tau=\tau_{0,2}^{1}}\right\}<0
$$

By Theorem 4.5, system (2.3) undergoes a Hopf bifurcation at $\tau=\tau_{0,1}^{1}$ and at $\tau=\tau_{0,2}^{1}$. For $\tau$ less than $\tau_{0,1}^{1}$, the equilibrium $E_{+}$is asymptotically stable (see Figure 15). For $\tau$ greater than $\tau_{0,1}^{1}$, 


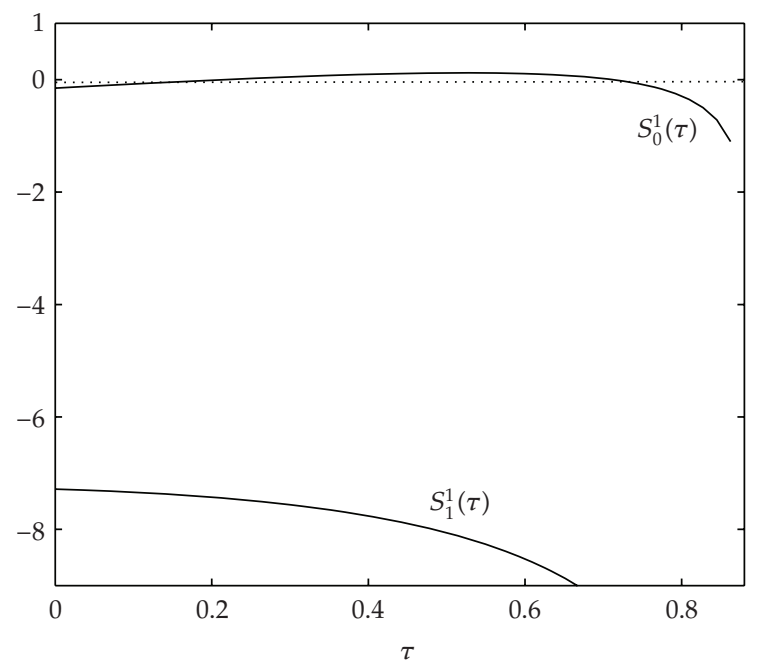

Figure 14: Verification of the transversality condition required for Hopf bifurcation. Parameters are the same as in Figure 13.

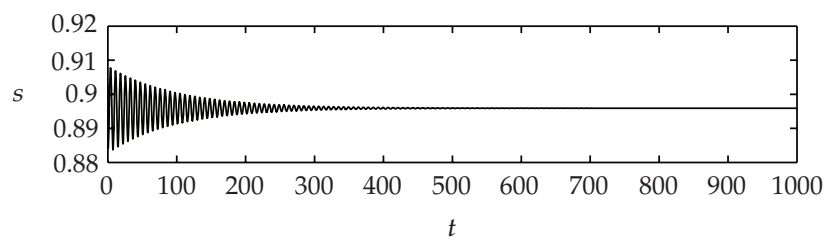

(a)

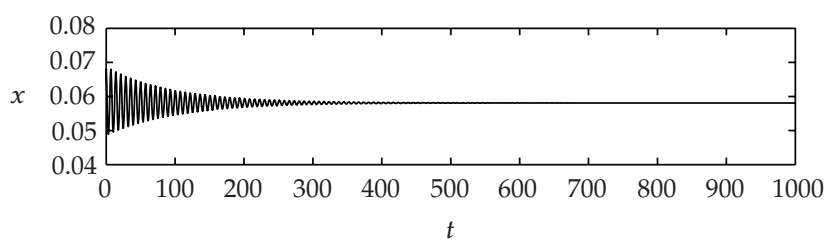

(b)

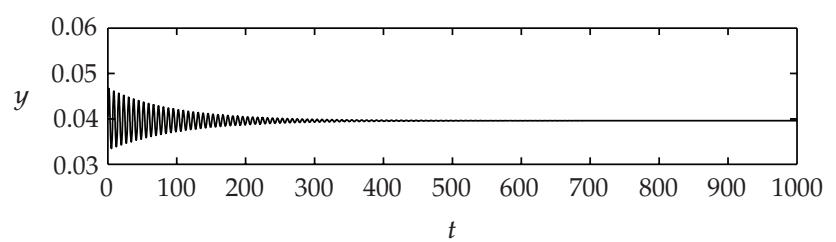

(c)

Figure 15: Equilibrium $E_{+}(\tau)$ is stable when $\tau=0.15<\tau_{0,1}^{1}$. The other parameters are the same as in Figure 13.

but less than $\tau_{0,2}^{1}$, there is an orbitally asymptotically stable periodic solution surrounding the equilibrium $E_{+}$(see Figures 16 and 17). At $\tau=\tau_{0,2}^{1}$, there is a second Hopf bifurcation, where 


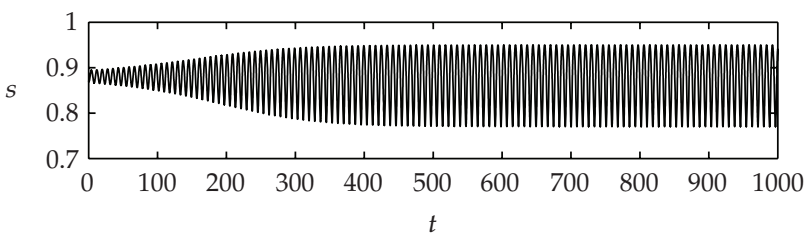

(a)

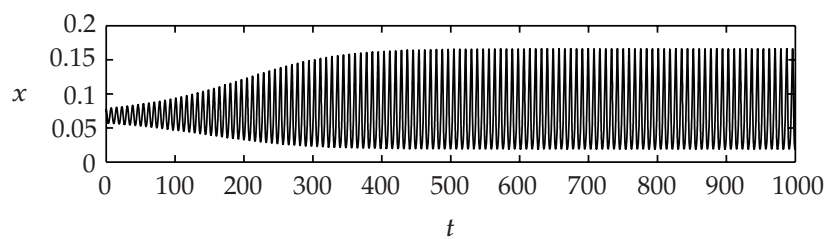

(b)

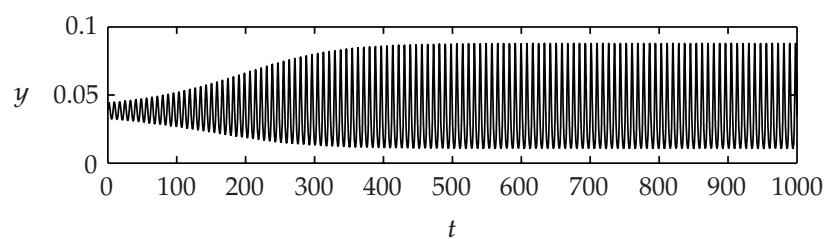

(c)

Figure 16: Time series of a solution with constant initial data $s(0)=0.87, x(t)=0.077$, and $y(t)=0.048$ for $t \in[-0.3,0]$, that approaches a stable periodic solution as time increases. In this figure, $\tau=0.3$. The other parameters are the same as in Figure 13.

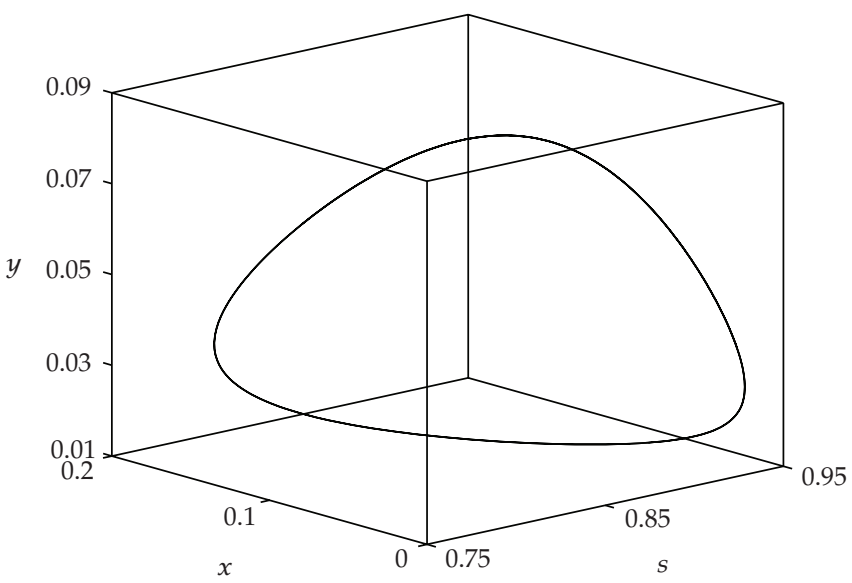

Figure 17: The attracting periodic solution shown in Figure 16 in phase space. Note that $\tau=0.3>\tau_{0,1}^{1}$.

the periodic solution coalesces with $E_{+}$. For $\tau>\tau_{0,2}^{1}$, the periodic orbit no longer exists and $E_{+}$ regains stability (see Figure 18) until it disappears when $\tau>\tau_{c}$. 


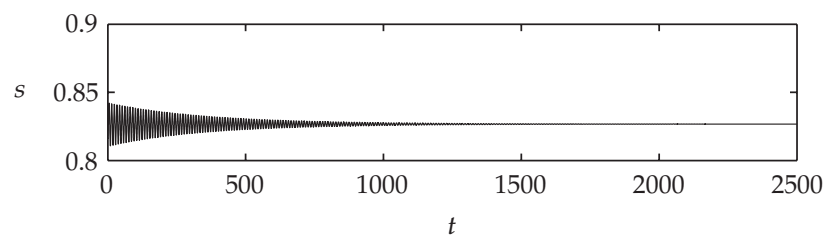

(a)

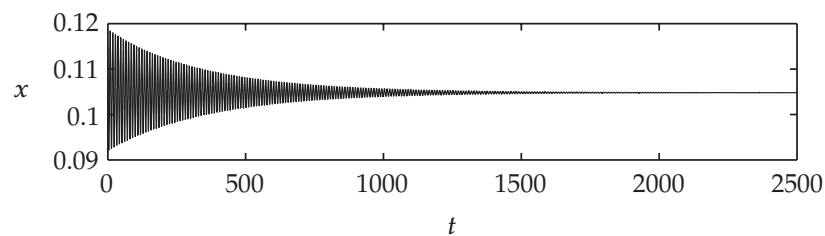

(b)

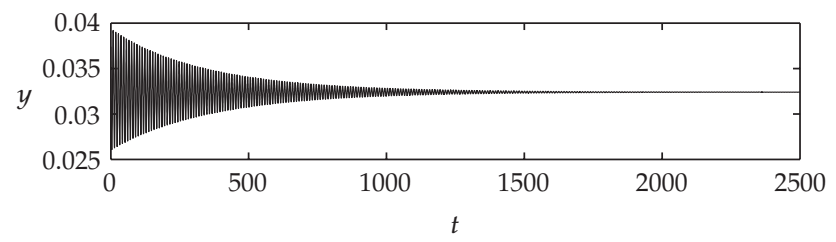

(c)

Figure 18: The periodic solution disappears and $E_{+}(\tau)$ regains its stability when $\tau>\tau_{0}^{2}$. In this figure, $\tau=0.74$. The other parameters are the same as in Figure 13 .

\section{Appendix}

\section{Preliminary Results}

To establish the existence of periodic solutions in autonomous delay differential equations, one of the simplest ways is through Hopf Bifurcation. Below is a general Hopf Bifurcation theorem for delay differential equations due to De Oliveira [14]. Before stating the theorem we require some notation.

Consider a one parameter family of neutral delay differential equations:

$$
\frac{\mathrm{d}}{\mathrm{d} t}\left[D\left(\alpha, x_{t}\right)-g\left(\alpha, x_{t}\right)\right]=L\left(\alpha, x_{t}\right)+f\left(\alpha, x_{t}\right), \quad \alpha \in \mathbb{R},
$$

where $D, L, f$, and $g$ are continuously differentiable in $\alpha$ and $x_{t} \in \mathbb{C}\left([-r, 0], \mathbb{R}^{n}\right)(r$ is a constant $), f(\alpha, 0)=g(\alpha, 0), \partial f(\alpha, 0) / \partial x_{t}=\partial g(\alpha, 0) / \partial x_{t}=0, D\left(\alpha, x_{t}\right)$ and $L\left(\alpha, x_{t}\right)$ are linear in $x_{t}$, and

$$
\begin{aligned}
& D\left(\alpha, x_{t}\right)=\sum_{k=0}^{\infty} A_{k}(\alpha) x\left(t-r_{k}(\alpha)\right)+\int_{-1}^{0} A(\alpha, \theta) x(t+\theta) \mathrm{d} \theta, \\
& L\left(\alpha, x_{\mathrm{t}}\right)=\sum_{k=0}^{\infty} A_{k}(\alpha) x\left(t-r_{k}(\alpha)\right)+\int_{-1}^{0} A(\alpha, \theta) x(t+\theta) \mathrm{d} \theta,
\end{aligned}
$$

for $x_{t} \in \mathbb{C}\left([-r, 0], \mathbb{R}^{n}\right)$. Assume $\alpha \in \mathbb{R}$, where $r_{0}(\alpha)=0, r_{k}(\alpha) \in(0,1]$, and $A_{k}(\alpha), B_{k}(\alpha)$, $A(\alpha, \theta)$, and $B(\alpha, \theta)$ satisfy 


$$
\sum_{k=0}^{\infty}\left(\left|A_{k}(\alpha)\right|+\left|B_{k}(\alpha)\right|\right)+\int_{-1}^{0}(|A(\alpha, \theta)|+|B(\alpha, \theta)|) \mathrm{d} \theta<\infty .
$$

It is easy to see that the characteristic matrix

$$
\Delta(\alpha, \lambda)=\lambda D\left(\alpha, e^{\lambda \cdot I} I\right)-L\left(\alpha, e^{\lambda \cdot I}\right)
$$

is continuously differentiable in $\alpha \in \mathbb{R}$ and $\Delta(\alpha, \lambda)$ is an entire function of $\lambda$. Making the following assumptions on (A.1).

$\left(S_{1}\right)$ There exist constants $a>0, b>0$ such that, for all complex values $\lambda$ such that $|\operatorname{Re}|<a$ and all $\alpha \in \mathbb{R}$, the following inequalities hold:

$$
\begin{gathered}
\left|\operatorname{det}\left(\sum_{k=0}^{\infty} A_{k}(\alpha) e^{-\lambda r_{k}(\alpha)}\right)\right| \geqslant b, \\
\left|\operatorname{det}\left(\sum_{k=0}^{\infty} A_{k}(\alpha) e^{-\lambda r_{k}(\alpha)}+\int_{-1}^{0} A(\alpha, \theta) e^{\lambda \theta} \mathrm{d} \theta\right)\right| \geqslant b .
\end{gathered}
$$

$\left(S_{2}\right)$ The characteristic equation $\operatorname{det} \Delta(\alpha, \lambda)=0$ has, for $\alpha=\alpha_{0}$, a simple purely imaginary root $\lambda_{0}=i v_{0}, v_{0}>0$, and no root of $\operatorname{det} \Delta\left(\alpha_{0}, \lambda\right)=0$, other than $\pm i v_{0}$, is an integral multiple of $\lambda_{0}$.

$\left(S_{3}\right) \operatorname{Re}\left(\partial \lambda\left(\alpha_{0}\right) / \partial \alpha\right) \neq 0$.

Now we are ready to state the Hopf bifurcation theorem for (A.1).

Theorem A.1 (Hopf bifurcation theorem, see Kuang [10, page 60]). In (A.1), assume that $\left(S_{1}\right)-\left(S_{3}\right)$ hold. Then there is an $\epsilon>0$ such that, for $a \in \mathbb{R},|a| \leqslant \epsilon$, there are functions $\alpha(a) \in \mathbb{R}$, $\omega(a) \in \mathbb{R}, \alpha(0)=\alpha_{0}, \omega(0)=2 \pi / v_{0}$, such that (A.1) has an $\omega(\alpha)$-periodic solution $x^{*}(a)(t)$, that is continuously differentiable in $t$, and a with $x^{*}(0)=0$. Furthermore, for $\left|\alpha-\alpha_{o}\right|<\epsilon,\left|\omega-\left(2 \pi / v_{0}\right)\right|<\epsilon$, every w-periodic solution $x(t)$ of (A.1) with $|x(t)|<\epsilon$ must be of this type, except for a translation in phase; that is, there exists $a \in(-\epsilon, \epsilon)$ and $b \in \mathbb{R}$ such that $x(t)=x^{*}(a)(t+b)$ for all $t \in \mathbb{R}$. et al. [15].

The following lemma is usually called the Fluctuation Lemma. For a proof, see Hirsh

Lemma A.2. Let $f: \mathbb{R}^{+} \rightarrow \mathbb{R}$ be a differentiable function. If $\lim _{\inf _{t \rightarrow \infty}} f(t)<\limsup _{t \rightarrow \infty} f(t)$, then there are sequences $t_{m} \uparrow \infty$ and $s_{m} \uparrow \infty$ such that for all $m$

$$
\begin{aligned}
& \dot{f}\left(t_{m}\right)=0, \quad f\left(t_{m}\right) \longrightarrow \lim \sup _{t \rightarrow \infty} f(t) \quad \text { as } m \longrightarrow \infty, \\
& \dot{f}\left(s_{m}\right)=0, \quad f\left(s_{m}\right) \longrightarrow \lim \inf _{t \rightarrow \infty} f(t) \quad \text { as } m \longrightarrow \infty,
\end{aligned}
$$

The proof of the following useful lemma can be found in [16]. 
Theorem A.3. Let $a \in(-\infty, \infty)$ and $f:[a, \infty) \rightarrow \mathbb{R}$ be a differentiable function. If $\lim _{t \rightarrow \infty} f(t)$ exists (finite) and the derivative function $\dot{f}(t)$ is uniformly continuous on $(a, \infty)$, then $\lim _{t \rightarrow \infty} \dot{f}(t)=0$.

\section{Acknowledgment}

This research is partially supported by NSERC.

\section{References}

[1] S. B. Hsu, S. Hubbell, and P. Waltman, "A mathematical theory for single-nutrient competition in continuous cultures of micro-organisms," SIAM Journal on Applied Mathematics, vol. 32, no. 2, pp. 366383, 1977.

[2] H. L. Smith and P. Waltman, The Theory of the Chemostat: Dynamics of Microbial Competition, vol. 13 of Cambridge Studies in Mathematical Biology, Cambridge University Press, Cambridge, UK, 1995.

[3] G. J. Butler, S. B. Hsu, and P. Waltman, "Coexistence of competing predators in a chemostat," Journal of Mathematical Biology, vol. 17, no. 2, pp. 133-151, 1983.

[4] Y. Kuang, "Limit cycles in a chemostat-related model," SIAM Journal on Applied Mathematics, vol. 49, no. 6, pp. 1759-1767, 1989.

[5] G. J. Butler and G. S. K. Wolkowicz, "Predator-mediated competition in the chemostat," Journal of Mathematical Biology, vol. 24, no. 2, pp. 167-191, 1986.

[6] G. S. K. Wolkowicz and H. Xia, "Global asymptotic behavior of a chemostat model with discrete delays," SIAM Journal on Applied Mathematics, vol. 57, no. 4, pp. 1019-1043, 1997.

[7] G. S. K. Wolkowicz, "Successful invasion of a food web in a chemostat," Mathematical Biosciences, vol. 93, no. 2, pp. 249-268, 1989.

[8] R. K. Miller and A. N. Michel, Ordinary Differential Equations, Academic Press, New York, NY, USA, 1982.

[9] R. Bellman and K. L. Cooke, Differential-Difference Equations, Academic Press, New York, NY, USA, 1963.

[10] Y. Kuang, Delay Differential Equations with Applications in Population Dynamics, vol. 191 of Mathematics in Science and Engineering, Academic Press, Boston, Mass, USA, 1993.

[11] K. Engelborghs, T. Luzyanina, and D. Roose, "Numerical bifurcation analysis of delay differential equations using DDE-BIFTOOL," Transactions on Mathematical Software, vol. 28, no. 1, pp. 1-21, 2002.

[12] K. Verheyden, "New functionality in dde-biftool v.2.03.," Addendum to the manual of DDE-BIFTOOL v. 2.00 (and v.2.02), unpublished, 2007.

[13] E. Beretta and Y. Kuang, "Geometric stability switch criteria in delay differential systems with delay dependent parameters," SIAM Journal on Mathematical Analysis, vol. 33, no. 5, pp. 1144-1165, 2002.

[14] J. C. F. De Oliveira, "Hopf bifurcation for functional differential equations," Nonlinear Analysis, vol. 4, no. 2, pp. 217-229, 1980.

[15] W. M. Hirsch, H. Hanisch, and J.-P. Gabriel, “Differential equation models of some parasitic infections: methods for the study of asymptotic behavior," Communications on Pure and Applied Mathematics, vol. 38, no. 6, pp. 733-753, 1985.

[16] K. Golpalsalmy, Stability and Oscillations in Delay Differential Equations of Population Dynamics, Kluwer Academic Publishers, Dordrecht, The Netherlands, 1985. 


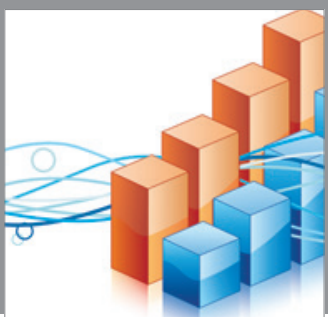

Advances in

Operations Research

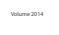

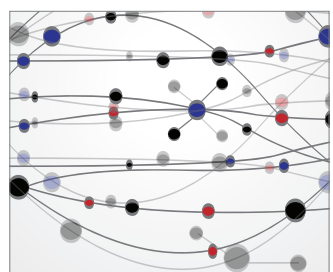

\section{The Scientific} World Journal
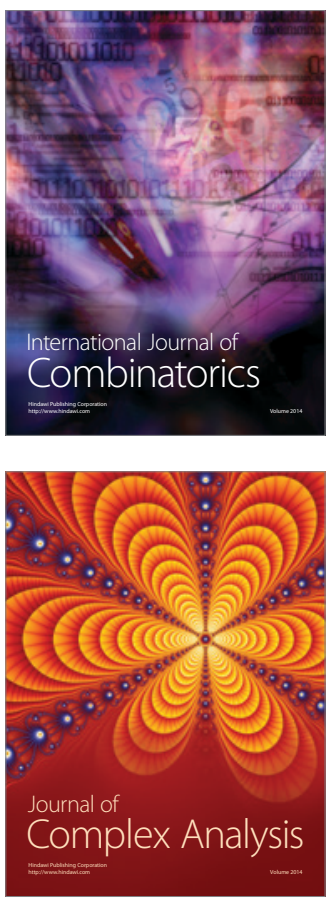

International Journal of

Mathematics and

Mathematical

Sciences
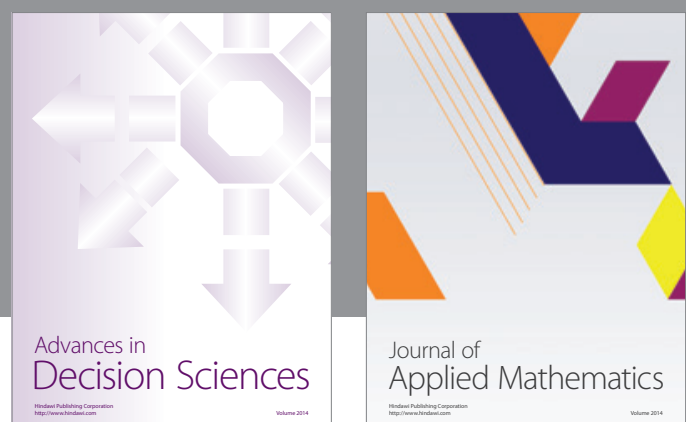

Journal of

Applied Mathematics
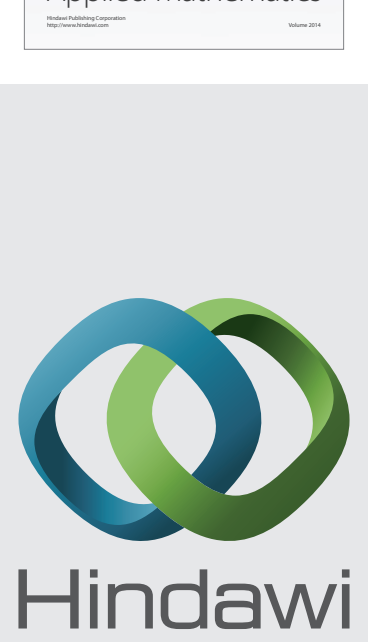

Submit your manuscripts at http://www.hindawi.com
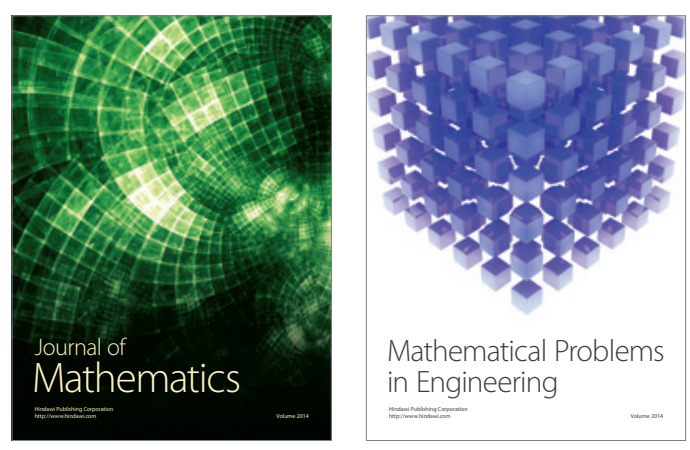

Mathematical Problems in Engineering
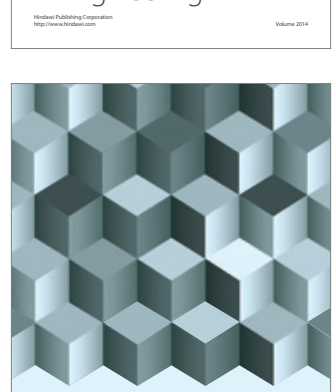

Journal of

Function Spaces
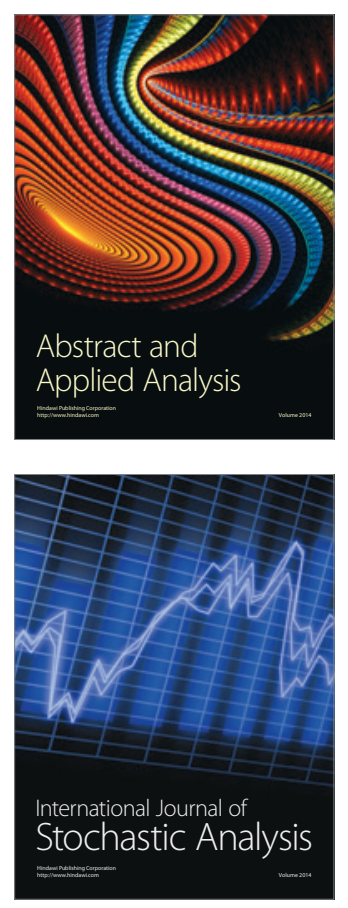

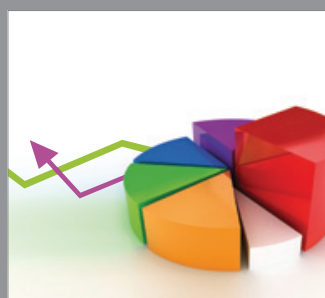

ournal of

Probability and Statistics

Promensencen
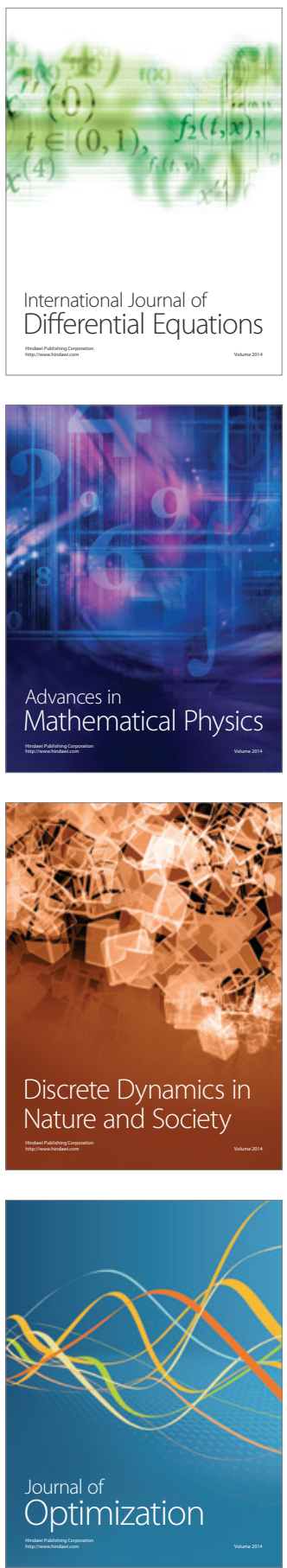